\title{
Population Dynamics of Spiking Neurons: Fast Transients, Asynchronous States, and Locking
}

\author{
Wulfram Gerstner \\ Center for Neuromimetic Systems, Swiss Federal Institute of Technology, EPFL-DI, \\ CH-1015 Lausanne, Switzerland
}

An integral equation describing the time evolution of the population activity in a homogeneous pool of spiking neurons of the integrate-and-fire type is discussed. It is analytically shown that transients from a state of incoherent firing can be immediate. The stability of incoherent firing is analyzed in terms of the noise level and transmission delay, and a bifurcation diagram is derived. The response of a population of noisy integrateand-fire neurons to an input current of small amplitude is calculated and characterized by a linear filter $\mathcal{L}$. The stability of perfectly synchronized "locked" solutions is analyzed.

\section{Introduction}

In many areas of the brain, neurons are organized in populations of units with similar properties. The most prominent examples are probably columns in the somatosensory and visual cortex (Hubel \& Wiesel, 1962; Mountcastle, 1957) and pools of motor neurons (Kandel \& Schwartz, 1991). In such a situation, we may want to describe the mean activity of the neuronal population rather than the spiking of individual neurons. In a population of $N$ neurons, we can formally determine the proportion of active neurons by counting the number of spikes $n_{\text {act }}(t ; t+\Delta t)$ in a small time interval $\Delta t$ and dividing by $N$. Division by $\Delta t$ yields the activity

$$
A(t)=\lim _{\Delta t \rightarrow 0} \frac{1}{\Delta t} \frac{n_{\mathrm{act}}(t ; t+\Delta t)}{N} .
$$

Although equation 1.1 has units of a rate, the concept of a population average is quite distinct from the definition of a firing rate via a temporal average. Temporal averaging over many spikes of a single neuron is a concept that works well if the input is constant or changes on a time scale that is slow with respect to the size of the temporal averaging window. Sensory input in a real-world scenario, however, is never constant. Moreover, reaction times are often short, which indicates that neurons do not have the time for temporal averaging (Thorpe, Fize, \& Marlot, 1996). The population activity, on the other hand, may react quickly to changes in the input 

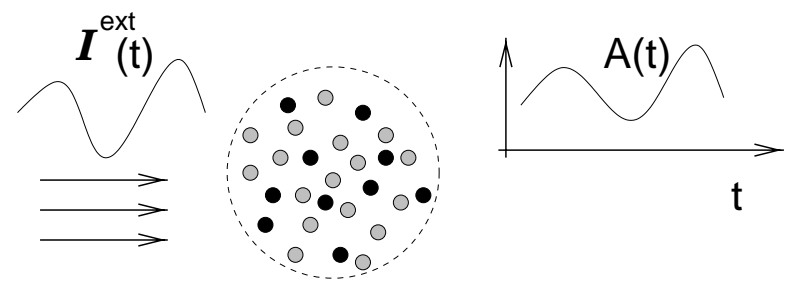

Figure 1: Population of neurons (schematic). All neurons receive the same input $\mathcal{I}^{\text {ext }}(t)$ (left), which results in a time-dependent population activity $A(t)$ (right).

(Knight, 1972a; Treves, 1992; Tsodyks \& Sejnowski, 1995; van Vreeswijk \& Sompolinsky, 1996).

In this article we study the properties of a large and homogeneous population of spiking neurons. All neurons are identical, receive the same input $\mathcal{I}^{\text {ext }}(t)$, and are mutually coupled by synapses of uniform strength (see Figure 1). Can we write down a dynamic equation in continuous time that describes the evolution of the population activity? This problem comes up in various models and has been studied by several researchers (Knight, 1972a; Wilson \& Cowan, 1972, 1973; Amari, 1974; Gerstner \& van Hemmen, 1992, 1993, 1994; Bauer \& Pawelzik, 1993; Gerstner, 1995; Pinto, Brumberg, Simons, \& Ermentrout, 1996; Senn et al., 1996; Eggert \& van Hemmen, 1997; Pham, Pakdamen, Champagnat, \& Vibert, 1998). A frequently adopted solution is a differential equation (Wilson \& Cowan, 1972),

$$
\tau \frac{d A(t)}{d t}=-A(t)+g\left(\int_{0}^{\infty} \epsilon(s) A(t-s)+\tilde{\epsilon}(s) \mathcal{I}^{\mathrm{ext}}(t-s) d s\right)
$$

where neurons are coupled to each other with a delay kernel $\epsilon$ and receive external input (or input from other populations) filtered by a kernel $\tilde{\epsilon} ; g($.$) is$ the transfer function of the population and $\tau$ is a time constant. Equation 1.2 is equivalent to the equations of graded-response neurons (Cowan, 1968; Cohen \& Grossberg, 1983; Hopfield, 1984).

Equation 1.2 introduces a time constant $\tau$ that is basically ad hoc. Wilson and Cowan $(1972,1973)$ have derived (1.2) from an integral equation,

$$
\begin{aligned}
A(t)=\left[1-\int_{0}^{\delta^{\mathrm{abs}}} A(t-s) d s\right] g\left(\int_{0}^{\infty} \epsilon(s) A(t-s)\right. \\
\left.+\tilde{\epsilon}(s) \mathcal{I}^{\mathrm{ext}}(t-s) d s\right),
\end{aligned}
$$

which is valid for stochastic neurons with an absolute refractory period of length $\delta^{\text {abs }}$. The term in the square brackets accounts for the fact that neurons that have fired between $t-\delta^{\text {abs }}$ and $t$ are in the refractory state and 
cannot fire. $g($.$) is the neuronal transfer function. Wilson and Cowan sub-$ sequently used a method of time-coarse graining to transform equation 1.3 into the differential equation 1.2. The time constant $\tau$, which appears on the left-hand side of equation 1.2, is related to the window of time-coarse graining.

It has been argued that $\tau$ in equation 1.2 is the membrane time constant of the neurons, an assertion that may be criticized since it holds for slowly changing activities only (Wilson \& Cowan, 1972, 1973; Abbott \& van Vreeswijk, 1993; Gerstner, 1995). The activity is, however, not always slowly changing. It has been shown previously that the population activity can react quasi instantaneously to abrupt changes in the input (Treves, 1992; Tsodyks \& Sejnowski, 1995; van Vreeswijk \& Sompolinsky, 1996). Transients in networks of nonleaky integrate-and-fire neurons can be very short (Hopfield \& Herz, 1995). A population of leaky integrate-and-fire units can follow a periodic drive up to very high frequencies (Knight, 1972a, 1972b). Moreover, homogeneous networks may be in an oscillatory state where all neurons fire at exactly the same time (Mirollo \& Strogatz, 1990; Gerstner \& van Hemmen, 1993; Gerstner, van Hemmen, \& Cowan, 1996; Somers \& Kopell, 1993; van Vreeswijk, Abbott, \& Ermentrout, 1994; Hansel, Mato, \& Meunier, 1995; Terman \& Wang, 1995). In this case, the population activity changes rapidly between zero and a very high activity. Rapid transients and perfectly synchronized oscillations are inconsistent with a differential equation of the form 1.2.

In this article we analyze the population dynamics and derive a dynamic equation that is exact in the limit of a large number of neurons. The relevant equation is a generalization of the integral equations of Wilson and Cowan (1972) and Knight (1972a) and has been discussed previously in Gerstner and van Hemmen (1994) and Gerstner (1995). The dynamic properties of the population activity can be analyzed directly on the level of the integral equation; there is no need to transform it into a differential equation. The population equation allows us to discuss the following four questions from a unified point of view:

How does a population of neurons react to a fast change in the input? We show that during the initial phase of the transient, the population activity reacts instantaneously (Treves, 1992; Tsodyks \& Sejnowski, 1995; van Vreeswijk \& Sompolinsky, 1996). Specifically, the initial phase of the transient reflects directly the form $\tilde{\epsilon}$ of the postsynaptic potential. The end of the transient, on the other hand, can be rather slow and depends on the noise level and the coupling. In the special case of noiseless uncoupled integrate-and-fire neurons, a new periodic state is reached as soon as every neuron has fired once.

What are the conditions for exact synchrony in the firing of all neurons? We show that neurons are "locked" together if firing occurs while the input potential is rising. The locking theorem in Gerstner et al. (1996) follows naturally from the integral equation. 
What are the conditions to make the neurons fire in an optimally asynchronous manner? We discuss why, without noise, asynchronous firing is almost always unstable (Abbott \& van Vreeswijk, 1993; Gerstner \& van Hemmen, 1993; Gerstner, 1995). We show that with a proper choice of the delays and a sufficient amount of noise, the state of asynchronous firing can be stabilized (Gerstner, 1995). A bifurcation diagram is determined in terms of the noise level and the transmission delay.

What is the response of an asynchronously firing population to an arbitrary timedependent input current? We show that at least in the low-noise regime, the population activity responds faithfully even to high-frequency components of the input current (Knight, 1972a, 1972b). The cutoff frequency of the system is therefore given by the time constant of the synaptic current rather than by the membrane time constant. For some noise models, the cut-off frequency in the high-noise regime is further reduced.

Theories of population activity have a fairly long history (Wilson and Cowan, 1972, 1973; Knight, 1972a; Amari, 1974; Feldman \& Cowan, 1975). Some researchers have formulated population equations as maps in discrete time for homogeneous (Gerstner and van Hemmen, 1992; Bauer \& Pawelzik, 1993) or inhomogeneous populations (Senn et al., 1996; Pham et al., 1998). Others have formulated continuity equations for the probability distribution of the membrane potential with the threshold as an absorbing boundary (Abbott \& van Vreeswijk, 1993; Treves, 1993; Brunel \& Hakim, 1998; Nykamp, Tranchina, Shapley, \& McLoughlin, 1998). The population equations discussed in this article are related to the integral equations of Wilson and Cowan (1972) and Knight (1972a). Section 3 will review the population equations in continuous time developed in detail in Gerstner and van Hemmen (1992, 1994) and Gerstner (1995). Sections 4 through 7 will apply the population equations to the questions of locking, fast transients, signal transmission, and asynchronous firing.

\section{Model}

2.1 Deterministic Threshold Model. Model neurons are described by the spike response model, a variant of the integrate-and-fire model (Gerstner, 1995; Gerstner et al., 1996). A neuron $i$ fires if its membrane potential $u_{i}$ hits the threshold $\vartheta$. The membrane potential is of the form

$$
u_{i}(t)=\eta\left(t-\hat{t}_{i}\right)+h_{\mathrm{PSP}}\left(t \mid \hat{t}_{i}\right),
$$

where $\hat{t}_{i}$ is the most recent firing time of neuron $i$, and

$$
\begin{aligned}
h_{\mathrm{PSP}}\left(t \mid \hat{t}_{i}\right)= & \sum_{j \in \Gamma_{i}} \sum_{t_{j}^{(f)}} w_{i j} \epsilon\left(t-\hat{t}_{i}, t-t_{j}^{(f)}\right) \\
& +J^{\mathrm{ext}} \int_{0}^{\infty} \tilde{\epsilon}\left(t-\hat{t}_{i}, s\right) \mathcal{I}^{\mathrm{ext}}(t-s) d s
\end{aligned}
$$


is the postsynaptic potential caused by firings $t_{j}^{(f)}$ of presynaptic neurons $j \in \Gamma_{i}$ or by external input $\mathcal{I}^{\text {ext }}(t)$. The kernel $\eta($.) in equation 2.1 is a (negative) contribution due to refractoriness. Alternatively, we could incorporate refractoriness into the threshold and define a dynamic threshold $\vartheta-\eta\left(t-\hat{t}_{i}\right)$.

Since we are interested in a homogeneous population of neurons, the kernels $\eta, \epsilon$, and $\tilde{\epsilon}$ have no indices $i, j$, and all neurons receive the same input $\mathcal{I}^{\text {ext }}(t)$. Moreover, the interaction strength between the neurons is uniform,

$$
w_{i j}=\frac{J_{0}}{N}
$$

where $J_{0}$ is a parameter. The interaction strength scales with one over the number $N$ of neurons so that the total input remains finite in the limit of $N \rightarrow \infty$.

The theoretical approach developed in this article is valid for arbitrary response kernels $\epsilon$ and $\tilde{\epsilon}$ and for a broad variety of refractory kernels $\eta$. Occasionally, however, we may want to specify the response kernels. For example, with an appropriate choice of the kernels $\epsilon, \tilde{\epsilon}$, and $\eta$, equation 2.1 gives an excellent approximation to the Hodgkin-Huxley model with timedependent input (Kistler, Gerstner, \& van Hemmen, 1997). Kernels can also be adjusted to experimental data (Stevens \& Zador, 1998). With a different choice of kernels, equation 2.1 can be mapped exactly to various versions of the integrate-and-fire model (Gerstner, 1995). Although most of the theory developed in this article is general, we will use two specific models for an illustration of the results.

2.1.1 Simple Spike Response Model $\left(S R M_{0}\right)$. In the first model, we assume that the kernels $\epsilon$ and $\tilde{\epsilon}$ do not depend on their first argument: $\epsilon(t-\hat{t}, s)=$ $\epsilon_{0}(s)$ and $\tilde{\epsilon}(t-\hat{t}, s)=\tilde{\epsilon}_{0}(s)$. This is the simplest instance of a spike response model (Gerstner \& van Hemmen, 1992) and will be called SRM 0 . In this case, the postsynaptic potential is independent of the last firing time of the neuron. We set $h_{\mathrm{PSP}}(t \mid \hat{t})=h(t)$ with

$$
h(t)=\sum_{j \in \Gamma_{i}} \sum_{t_{j}^{(f)}} w_{i j} \epsilon_{0}\left(t-t_{j}^{(f)}\right)+J^{\mathrm{ext}} \int_{0}^{\infty} \tilde{\epsilon}_{0}(s) \mathcal{I}^{\mathrm{ext}}(t-s) d s .
$$

We will refer to $h(t)$ as the input potential. In simulations we need to specifiy the kernels. We take

$$
\epsilon_{0}(s)=\frac{s-\Delta^{\mathrm{ax}}}{\tau^{2}} \exp \left(-\frac{s-\Delta^{\mathrm{ax}}}{\tau}\right) \mathcal{H}\left(s-\Delta^{\mathrm{ax}}\right),
$$

where $\Delta^{\mathrm{ax}}$ is the axonal delay and $\tau$ is a membrane time constant. The normalization is $\int \epsilon_{0}(s) d s=1$. As always, $\mathcal{H}(s)$ is the Heaviside step function 
with $\mathcal{H}(s)=0$ for $s \leq 0$ and $\mathcal{H}(s)=1$ for $s>0$. For external input we use in simulations

$$
\tilde{\epsilon}_{0}(s)=\frac{1}{\tau} \exp \left(-\frac{s}{\tau}\right) \mathcal{H}(s)
$$

with the same time constant $\tau$ as in equation 2.5. After each spike the membrane potential is reset by adding a refractory kernel,

$$
\eta(s)=-\tilde{\eta}_{0} \exp (-s / \tau) \mathcal{H}(s) .
$$

In simulations of $\mathrm{SRM}_{0}$ neurons we set $\tilde{\eta}_{0}=1$. The spike response model with the specific kernels defined in equations 2.5 through 2.7 , can be considered as an approximation to the integrate-and-fire model. The approximation is based on a truncation and is valid if the typical interspike interval $T_{0}$ is much larger than the time constant $\tau$ (Gerstner et al., 1996); the error of the approximation is of the order $\exp \left(-T_{0} / \tau\right)$. For the numerical analysis later on, we have adjusted the threshold $\vartheta$ so that the mean interspike interval $T_{0}$ is exactly $T_{0}=2 \tau$. In this case, we may expect that $S_{R M}$ gives a reasonable, albeit not perfect, approximation to the integrate-and-fire model. A major advantage of $\mathrm{SRM}_{0}$ neurons (compared to integrate-and-fire neurons) is that most of the results of sections 3 through 7 can be formulated in a mathematically transparent form, which allows an easy interpretation.

2.1.2 Integrate-and-Fire model (IF). With a different choice of the kernels $\epsilon$ and $\tilde{\epsilon}$ in equation 2.2, it is possible to construct a perfect mapping between equation 2.1 and the standard integrate-and-fire model (Gerstner, 1995). Between two firings, the membrane potential of an integrate-and-fire (IF) unit $i$ changes according to

$$
\tau_{m} \frac{d u_{i}}{d t}=-u_{i}+\sum_{j} \sum_{t_{j}^{(f)}} w_{i j} \alpha\left(t-t_{j}^{(f)}\right)+J^{\text {ext }} \mathcal{I}^{\text {ext }}(t),
$$

where $\tau_{m}$ is the membrane time constant, $w_{i j}$ is the coupling strength, and $\alpha($.$) is the time course of the synaptic current. If u_{i}$ hits a threshold $\vartheta$, the membrane potential is reset to $u_{\text {reset }}<\vartheta$. The spike response equation, 2.1, can be considered an integrated version of equation 2.8. The value $u_{\text {reset }}$ comes in as an initial condition for the integration of equation 2.8 and leads to a kernel $\eta$ as in equation 2.7 with $\tau=\tau_{m}$ and $\tilde{\eta}_{0}=-u_{\text {reset. }}$. For $u_{\text {reset }}=0$, the $\eta$-kernel vanishes. Integration of the synaptic current terms in equation 2.8 yields

$$
\begin{aligned}
\epsilon(x, s) & =\int_{s-x}^{s} \alpha\left(s^{\prime}\right) e^{-\left(s-s^{\prime}\right) / \tau_{m}} d s^{\prime} \\
& =\epsilon_{0}(s)-\epsilon_{0}(s-x) e^{-x / \tau_{m}},
\end{aligned}
$$


where $\epsilon_{0}(s)=\int_{0}^{s} d s^{\prime} \alpha\left(s^{\prime}\right) \exp \left[-\left(s-s^{\prime}\right) / \tau_{m}\right]$. The response kernel defined in equation 2.5 corresponds to a synaptic current $\alpha(s)=\tau_{s}^{-1} \exp \left(-s / \tau_{s}\right) \mathcal{H}(s)$ with time constant $\tau_{s}=\tau_{m}=\tau$ and an additional axonal transmission delay $\Delta^{\text {ax }}$. Similarly, $\tilde{\epsilon}(x, s)=\tilde{\epsilon}_{0}(s)-\tilde{\epsilon}_{0}(s-x) e^{-x / \tau_{m}}$ with $\tilde{\epsilon}_{0}$ given by equation 2.6 and $\tau_{m}=\tau$. Using the expressions for $\tilde{\epsilon}$ and $\epsilon$ with $\tau_{m}=\tau$, the postsynaptic potential for IF units can be expressed as

$$
h_{\mathrm{PSP}}(t \mid \hat{t})=h(t)-h(\hat{t}) e^{-(t-\hat{t}) / \tau},
$$

where the input potential $h(t)$ is given by equation 2.4 . We emphasize that the specific choice of the kernel $\epsilon_{0}$ in equation 2.5 corresponds to a synaptic current with fast rise time. The theoretical framework of this article, however, is more general and applies equally well to slowly rising synaptic currents.

2.2 Noise. The neuron model discussed so far is purely deterministic. Given a spike at $\hat{t}$ and for a known postsynaptic potential $h_{\mathrm{PSP}}(t \mid \hat{t})$, we can calculate the interval until the next spike from the threshold condition,

$$
T(\hat{t})=\min \{(t-\hat{t}) \mid u(t)=\vartheta ; t>\hat{t}\} .
$$

In words, the interval $T$ is given by the first threshold crossing after the spike at $\hat{t}$.

In the presence of noise, the exact firing time and, hence, the exact interval are unknown. We may, however, determine the probability density $P_{h}(t \mid \hat{t})$ that firing occurs around time $t$ given the postsynaptic potential $h_{\mathrm{PSP}}\left(t^{\prime} \mid \hat{t}\right)$ for $\hat{t}<t^{\prime} \leq t$ and knowing the last firing time $\hat{t}$. The distribution $P_{h}(t \mid \hat{t})$ will play an important role for the population equations in section 3 . In the noiseless case, the interval distribution reduces to a Dirac $\delta$-function

$$
P_{h}(t \mid \hat{t})=\delta[t-\hat{t}-T(\hat{t})]
$$

where $T(\hat{t})$ is the next interval given a spike at $\hat{t}$ and determined implicitly by equation 2.11 .

To calculate $P_{h}(t \mid \hat{t})$ in the noisy case, we have to decide on a noise model. There are at least three different ways to include noise in spiking neurons. A popular procedure is to add white noise on the right-hand side of the differential equation of the IF neuron. Below this is called noise model C. Calculation of $P_{h}(t \mid \hat{t})$ requires the solution of a first passage time problem, which is known to be hard (Tuckwell, 1988, 1989). It is therefore convenient to work with one of the following two noise models: threshold noise (noise model A) or reset noise (noise model B).

2.2.1 Noisy Threshold (Noise Model A). In this first noise model, we assume that the neuron can fire even though the formal threshold $\vartheta$ has not 
been reached yet or may stay quiescent even though the membrane potential is above threshold. To do this consistently, we introduce an "escape rate $^{\prime \prime} \rho$, which depends on the distance between the momentary value of the membrane potential and the threshold,

$$
\rho(t)=f[u(t)-\vartheta]
$$

In the mathematical literature, the quantity $\rho$ would be called a stochastic intensity. We require $f(x) \rightarrow 0$ for $x \rightarrow-\infty$. Otherwise the choice of the function $f$ is arbitrary. Plausible assumptions are an exponential dependence $\rho=\rho_{0} \exp [\beta(u-\vartheta)]$ or a gaussian dependence $\rho=\rho_{0} \exp \left[-\beta(u-\vartheta)^{2}\right]$; or a step function $\rho=\rho_{0} \mathcal{H}(u-\vartheta)$; or a piecewise linear function $\rho=\rho_{0}(u-$ $\vartheta) \mathcal{H}(u-\vartheta) . \beta$ and $\rho_{0}$ are parameters. Note that the escape rate $\rho$ is implicitly time dependent, since the membrane potential $u(t)=\eta(t-\hat{t})+h_{\mathrm{PSP}}(t \mid \hat{t})$ varies over time. In addition, we may also include an explicit time dependence, for example, to account for a reduced spiking probability immediately after the spike at $\hat{t}$.

Let us now calculate $P_{h}(t \mid \hat{t})$, the probability density of having a spike at $t$ given that the last spike occurred at $\hat{t}$, and in the presence of a postsynaptic potential $h_{\mathrm{PSP}}(t \mid \hat{t})$ for $t>\hat{t}$. At each moment of time, the value $u(t)$ of the membrane potential determines the escape rate $\rho(t)=f[u(t)-\vartheta]$. In order to emit the next spike at $t$, the neuron has to survive the interval $(\hat{t}, t)$ without firing and then fire at $t$. Given the escape rate $\rho(t)$, the probability of survival from $\hat{t}$ to $t$ without firing is

$$
S_{h}(t \mid \hat{t})=\exp \left(-\int_{\hat{t}}^{t} \rho\left(t^{\prime}\right) d t^{\prime}\right) .
$$

The probability density of firing at time $t$ is $\rho(t)$; thus with equation 2.14 we have

$$
P_{h}(t \mid \hat{t})=\rho(t) \exp \left(-\int_{\hat{t}}^{t} \rho\left(t^{\prime}\right) d t^{\prime}\right),
$$

which is the desired result. More detailed derivations of equation 2.15 can be found in Gerstner and van Hemmen (1994) and in the appendix of Wilson and Cowan (1972) (see also Cox \& Lewis, 1966)

2.2.2 Noisy Reset (Noise Model B). In this noise model, firing is given by the exact threshold condition $u(t)=\vartheta$. Noise is included in the formulation of reset and refractoriness. A convenient way of doing this is by replacing $\tilde{\eta}_{0}$ in equation 2.7 by

$$
\tilde{\eta}_{0} \longrightarrow \eta_{0}(r)=\tilde{\eta}_{0} e^{r / \tau},
$$


where $r$ is a random variable with zero mean. In the language of the IF neuron, we can describe the effect of $r$ as a stochastic component in the value of the reset potential. After each spike, a new value of $r$ is drawn randomly from a gaussian distribution $\mathcal{G}_{\sigma}(r)$ with variance $\sigma \ll \tau$. Let us write $T(\hat{t}, r)$ for the next interval of a neuron that has fired at $\hat{t}$ and was reset with a stochastic value $r$. Since $r$ is drawn at random, the interval distribution is

$$
P_{h}(t \mid \hat{t})=\int d r \delta[t-\hat{t}-T(\hat{t}, r)] \mathcal{G}_{\sigma}(r)
$$

In order to keep the arguments simple, we assume in the following that the typical interval $T_{0}(\hat{t})=T(\hat{t}, 0)$ is significantly larger than the width $\sigma$ of the distribution.

In the case of the simple spike response model $\mathrm{SRM}_{0}$, a reset according to equation 2.16 with $r \neq 0$ shifts the refractory kernel horizontally along the time axis. To see this, let us consider a neuron that has fired its last spike at $\hat{t}$ and has been reset with variable $r$. Its refractory kernel is $\eta(t)=$ $\tilde{\eta}_{0} \exp [-(t-\hat{t}-r) / \tau]$. Thus the neuron acts like a noiseless neuron that has fired its last spike at $t^{\prime}=\hat{t}+r$. We therefore have $T(\hat{t}, r)=r+T_{0}(\hat{t}+r)$, where $T_{0}\left(t^{\prime}\right)$ is the interval of a noiseless neuron that has fired at $t^{\prime}$. For a constant input potential $h(t)=h_{0}$, the interval distribution of a SRM $\mathrm{SR}_{0}$ neuron is therefore a gaussian distribution centered at the noise-free interval $T_{0}$, $P_{h_{0}}(t \mid \hat{t})=\mathcal{G}_{\hat{\sigma}}\left(t-\hat{t}-T_{0}\right)$ with variance $\hat{\sigma}=\sigma$. Thus, the gaussian distribution $\mathcal{G}_{\sigma}(r)$ of the noise variable $r$ maps directly to a gaussian distribution of the intervals around the mean $T_{0}$.

In the case of the IF neuron, the argument is slightly more involved. For $\sigma \ll T_{0}(\hat{t})$ we may expand the interval $T(\hat{t}, r)=T_{0}(\hat{t})+r T_{1}(\hat{t})+\mathcal{O}\left(r^{2}\right)$ in equation 2.17 to linear order in $r$. From the threshold condition, equation 2.11, we find $T_{1}(\hat{t})=\eta^{\prime} / u^{\prime}$ where $\eta^{\prime}$ denotes the derivative of $\eta(s)$ at $s=T_{0}(\hat{t})$ and $u^{\prime}=d u / d t$ is to be evaluated at $t=\hat{t}+T_{0}(\hat{t})$. The integration on the right-hand side of equation 2.17 can now be performed and yields

$$
P_{h}(t \mid \hat{t})=\mathcal{G}_{\hat{\sigma}}\left[t-\hat{t}-T_{0}(\hat{t})\right]
$$

with variance $\hat{\sigma}=\sigma T_{1}(\hat{t})$. Hence the interval distribution is a gaussian around the noise-free interval. For a constant input potential $h_{0}$, we find from equation $2.10 T_{1}=\tilde{\eta}_{0} /\left(\tilde{\eta}_{0}+h_{0}\right)$, which may be used for the calculation of $\hat{\sigma}$.

2.2.3 Noisy Integration (Noise Model C). A noise term is added on the right-hand side of equation 2.8 (Tuckwell, 1988, 1989),

$$
\tau_{m} \frac{d u_{i}}{d t}=-u_{i}+\sum_{j} \sum_{t_{j}^{(f)}} w_{i j} \alpha\left(t-t_{j}^{(f)}\right)+J^{\mathrm{ext}} \mathcal{I}^{\mathrm{ext}}(t)+\xi
$$


where $\xi$ represents gaussian white noise with zero mean and correlation $\left\langle\xi(t) \xi\left(t^{\prime}\right)\right\rangle=D \tau_{m} \delta\left(t-t^{\prime}\right)$. The noise term can be motivated either by random input from background neurons that are not modeled explicitly (Stein, 1967) or by random openings of membrane channels. Noise causes the actual trajectory to drift away from the noise-free reference trajectory. In the absence of a threshold, the actual trajectories would have a gaussian distribution around the reference trajectory with variance $\sigma_{u}^{2}=(D / 2)\left[1-\exp \left(-2 t / \tau_{m}\right)\right]$ (Tuckwell, 1989). The threshold acts as an absorbing boundary, and $P_{h}(t \mid \hat{t})$ is the distribution of first passage times. In general, the first passage time problem is hard to solve. Even for constant driving current, the interval distribution is not symmetric around its mean, and higher moments are important. If, however, the level of noise is low and the reference trajectory hits the threshold with finite slope $u^{\prime}$, then a gaussian approximation of the distribution is reasonable (Tuckwell, 1989). In this case, the interval distribution is the same as for noise model B with $\sigma=\sigma_{u} / u^{\prime}$. On the other hand, if the reference trajectory stays significantly below threshold, then noise model $\mathrm{C}$ may be approximated by a noisy threshold (noise model $\mathrm{A}$ ) with escape rate $\alpha \rho_{0} \exp \left[-\beta(u-\vartheta)^{2}\right]$ with parameters $\rho_{0} \approx 1 / \tau_{m}$ and $\beta=D^{-1}$. (Plesser \& Gerstner, 1999). To avoid the complications of noise model C, we work in the following mainly with noise models A and B, except for some statements concerning C. A disadvantage of noise model B is that it generates spikes only for suprathreshold stimuli and cannot describe noise-activated spiking with subthreshold input. On the other hand, an indirect justification of noise model B could come from the theory of excitable membranes (Gutkin \& Ermentrout, 1998).

\section{Population Activity Equation}

We consider a homogeneous and fully connected network of spiking neurons in the limit of $N \rightarrow \infty$. We aim for a dynamic equation that describes the evolution of the population activity over time. The total number of spikes $n_{\text {act }}$ emitted by all neurons in the population is

$$
n_{\mathrm{act}}(t ; t+\Delta t)=\sum_{i=1}^{N} \sum_{t_{i}^{(f)}} \int_{t}^{t+\Delta t} \delta\left(t^{\prime}-t_{i}^{(f)}\right) d t^{\prime} .
$$

The population activity has already been defined in equation 1.1. Using equation 3.1 in equation 1.1, we may write

$$
A(t) d t=\frac{1}{N} \sum_{i=1}^{N} \delta\left(t-t_{i}^{(f)}\right) d t .
$$


The definition of $A$ allows us to rewrite the postsynaptic potential $h_{\mathrm{PSP}}$ in equation 2.2 in the form

$$
\begin{aligned}
h_{\mathrm{PSP}}(t \mid \hat{t})= & J_{0} \int_{0}^{\infty} \epsilon(t-\hat{t}, s) A(t-s) d s \\
& +J^{\mathrm{ext}} \int_{0}^{\infty} \tilde{\epsilon}(t-\hat{t}, s) \mathcal{I}^{\mathrm{ext}}(t-s) d s .
\end{aligned}
$$

Thus, given the activity $A\left(t^{\prime}\right)$ for $t^{\prime}<t$, we can determine the potential $h_{\mathrm{PSP}}(t)$ of a neuron that has fired its last spike at $\hat{t}$. What we need is another equation that allows us to determine the present activity $A(t)$ given the past. The equation for the activity dynamics will be derived from three observations:

1. The model neurons are supposed to show no adaptation. According to equation 2.1, the state of neuron $i$ depends explicitly on only the most recent firing time $\hat{t}_{i}$ (and, of course, on the input $h_{\mathrm{PSP}}$ ), but not on the firing times of earlier spikes of neuron $i$. This allows us to work in the framework of a (nonstationary) renewal theory (Cox, 1962; Gerstner, 1995).

2. In the limit of $N$ to infinity, only the expectation value of a random variable matters. The exact form of a probability distribution is irrelevant, and we can work directly with the mean. Full connectivity and $N \rightarrow \infty$ is the limit where mean-field theory becomes exact.

3. The total number of neurons in the population remains constant. We may exploit this fact to derive a conservation law.

3.1 Integral Equation for the Dynamics. Because of the first observation above, the probability density for a spike at $t$ depends only on $\hat{t}$ and on $h_{\mathrm{PSP}}(t \mid \hat{t})$ for $t>\hat{t}$. We have already used this knowledge in our notation $P_{h}(t \mid \hat{t})$ for the probability density of firing. The subscript $h$ stands for the dependence on $h_{\mathrm{PSP}}(t \mid \hat{t})$. Integration of the probability density over time $\int_{\hat{t}}^{t} P_{h}(s \mid \hat{t}) d s$ gives the probability that a neuron that has fired at $\hat{t}$ fires its next spike at some arbitrary time between $\hat{t}$ and $t$. Consequently, we can define a survival probability

$$
S_{h}(t \mid \hat{t})=1-\int_{\hat{t}}^{t} P_{h}(s \mid \hat{t}) d s,
$$

that is, the probability that a neuron that is under the influence of $h_{\mathrm{PSP}}$ and has fired its last spike at $\hat{t}$ survives without firing up to time $t$.

We now turn to a homogeneous population of neurons in the limit of $N \rightarrow \infty$. We consider the network state at time $t$. Because of the second ob- 
servation, the proportion of neurons that have fired their last spike between $t_{0}$ and $t$ (and have not fired since) is exactly

$$
\int_{t_{0}}^{t} S_{h}(t \mid \hat{t}) A(\hat{t}) d \hat{t}
$$

All neurons have fired at some point in the past; ${ }^{1}$ thus,

$$
\int_{-\infty}^{t} S_{h}(t \mid \hat{t}) A(\hat{t}) d \hat{t}=1
$$

Because of the third observation, the normalization equation 3.6 must hold at any arbitrary time $t$. Taking the derivative of equation 3.6 with respect to $t$ yields the activity dynamics,

$$
A(t)=\int_{-\infty}^{t} P_{h}(t \mid \hat{t}) A(\hat{t}) d \hat{t} .
$$

A different derivation of equation 3.7 is given in Gerstner and van Hemmen $(1992,1994)$ and Gerstner $(1993,1995)$.

The population equation 3.7 , with $h_{\mathrm{PSP}}$ given by 3.3 , is the starting point for the discussions in the following sections. Intuitively, equation 3.7 is easy to understand. The kernel $P_{h}(t \mid \hat{t})$ is the probability density that the next spike of a neuron under the influence of a potential $h_{\mathrm{PSP}}(t \mid \hat{t})$ occurs at time $t$ given that its last spike was at $\hat{t}$. The number of neurons that have fired at $\hat{t}$ is proportional to $A(\hat{t})$, and the integral runs over all the past.

An important remark concerns the proper normalization of the activity. Since equation 3.7 is defined as the derivative of equation 3.6, the integration constant on the right-hand side of the equation is lost. The system of equations 3.7 and 3.3 is therefore invariant under a rescaling of the activity $A \longrightarrow c A$ and $J_{0} \longrightarrow c^{-1} J_{0}$ with some constant $c$. To get the correct normalization, we have to go back to equation 3.6. In the following sections the population equations in the form of equations 3.7 or 3.6 are analyzed for various scenarios.

Equation 3.7 with the kernel $P_{h}(t \mid \hat{t})$ calculated for the potential $h_{\mathrm{PSP}}$ given by equation 3.3 defines the dynamics in a homogeneous network of spiking neurons with short-term memory. We remark that although equation 3.7 looks linear, it is in fact a highly nonlinear equation because the kernel $P_{h}(t \mid \hat{t})$ depends nonlinearly on $h_{\mathrm{PSP}}$, and $h_{\mathrm{PSP}}$ in turn depends again on the activity via equation 3.3. (See noise model $\mathrm{A}$ in section 2.2 for an explicit example of the kernel $P_{h}(t \mid \hat{t})$.)

\footnotetext{
${ }^{1}$ Neurons that have never fired before are assigned a formal firing time $\hat{t}=-\infty$
} 
3.2 Relation to the Wilson-Cowan Integral Equation. The WilsonCowan integral equation, 1.3 , is a special case of the population dynamics, equation 3.7. We assume neurons of the $\mathrm{SRM}_{0}$ type, $h_{\mathrm{PSP}}(t \mid \hat{t})=h(t)$, and consider noise model $\mathrm{A}$. The interval distribution is equation 2.15 with instantaneous rate $\rho(t)=f[u(t)]$. We may identify $f$ with the gain function $g$ of the Wilson-Cowan model. As shown in appendix A, for a refractory kernel $\eta$ with absolute refractoriness only, equation 3.7 reduces strictly to the Wilson-Cowan equation, 1.3. Moreover, the framework of the population equation 3.7 also works for relative refractoriness. In the appendix it is shown that equation 3.7 for $\mathrm{SRM}_{0}$ neurons with noise model A is formally equivalent to the population equations for neurons with relative refractoriness derived in the appendix of Wilson and Cowan (1972).

There is, however, an important difference in the interpretation of the equations. Wilson and Cowan motivated their gain function $g$ by a distribution of threshold values $\vartheta$ in an inhomogeneous population. In this case, the population equation 1.3 is an approximation, since correlations are neglected. In general, it matters whether the neurons in the refractory state are those with high or low threshold (Wilson \& Cowan, 1972). In our interpretation $g$ is the instantaneous escape rate due to a noisy threshold in a homogeneous population. In this interpretation, equation 1.3 is the exact equation for neurons with absolute refractoriness.

\section{Noise-Free Population Dynamics}

4.1 General Results. We start with a discussion of equation 3.7 in the noise-free case. As we have seen in equation 2.12 , the probability density $P_{h}(t \mid \hat{t})$ reduces in the limit of no noise to a Dirac $\delta$-function. We use equation 2.12 in 3.7 and find

$$
A(t)=\int_{-\infty}^{t} \delta(t-\hat{t}-T(\hat{t})) A(\hat{t}) d \hat{t} .
$$

Here $T(\hat{t})$ is the interval given implicitly by the threshold condition, equation 2.11. Note that $T(\hat{t})$ is the interval starting at $\hat{t}$ and looking forward toward the next spike.

The integration over the $\delta$-function in equation 4.1 can be done. Since $T$ in the argument of the $\delta$-function depends on $\hat{t}$, the result is

$$
A(t)=\left[1+\frac{\partial_{t} h+\partial_{\hat{t}} h}{\eta^{\prime}-\partial_{\hat{t}} h}\right]_{+} A\left[t-T_{b}(t)\right],
$$

where $T_{b}(t)$ is the backward interval given a spike at time $t$. The partial derivatives in equation $4.2, \partial_{t} h=d h_{\mathrm{PSP}}(t \mid \hat{t}) / d t$ and $\partial_{\hat{t}} h=d h_{\mathrm{PSP}}(t \mid \hat{t}) / d \hat{t}$, are to be evaluated at $\hat{t}=t-T_{b}(t)$. The derivative $\eta^{\prime}=d \eta / d s$ is to be evaluated at 


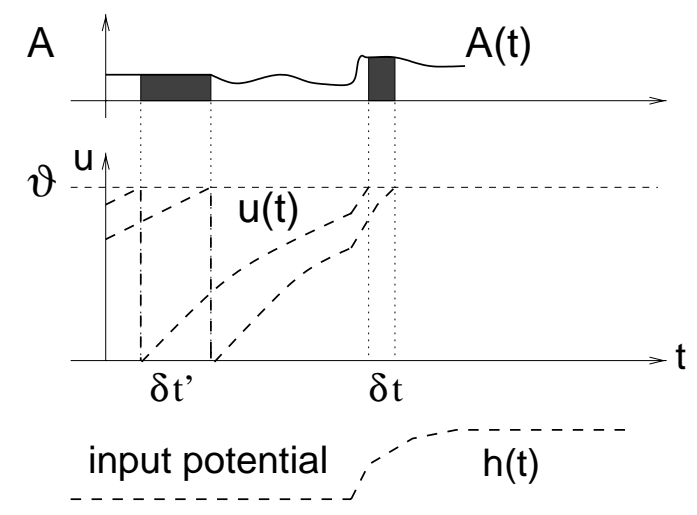

Figure 2: A change in the input potential $h$ with positive slope $h^{\prime}>0$ (dashed line, bottom) shifts neuronal firing times closer together (middle). As a result, the activity $A(t)$ (solid line, top) is higher at $t=\hat{t}+T(\hat{t})$ than it was at time $\hat{t}$ (schematic diagram).

$s=T_{b}(t)$. Note that the expression in the square brackets can also be written as $u^{\prime} /\left(\eta^{\prime}+\partial_{\hat{t}} h\right)$ with $u^{\prime}=d u /\left.d t\right|_{t}$ evaluated at $t$. Since the threshold must be reached from below, a spike at time $t$ is possible only if $u^{\prime}>0$. For $u^{\prime}<0$, the backward interval does not exist, and the activity vanishes. We have taken care of this fact by the notation $[x]_{+}=x$ for $x \geq 0$ and zero otherwise.

Equation 4.2 is the first major result of our analysis. In order to facilitate the discussion, we define a variable:

$$
C(t)=\frac{\partial_{t} h+\partial_{\hat{t}} h}{\eta^{\prime}-\partial_{\hat{t}} h} .
$$

Let us evaluate $C$ for $\mathrm{SRM}_{0}$ and IF-neurons.

For $\mathrm{SRM}_{0}$ neurons, all terms in equation 4.2 can be calculated explicitly. Since $\partial_{\hat{t}} h$ vanishes, equation 4.3 reduces to

$$
C^{\mathrm{SRM}}(t)=\frac{h^{\prime}(t)}{\eta^{\prime}\left[T_{b}(t)\right]} .
$$

The backward interval $T_{b}$ exists if $h(t)>\vartheta$ and is found from the threshold condition, equation 2.11, viz. $T_{b}(t)=\tau \ln \left\{\eta_{0} /[h(t)-\vartheta]\right\}$. With equation 4.4, the factor in square brackets in equation 4.2 becomes $\left[1+h^{\prime} / \eta^{\prime}\right]_{+}$. Its meaning is illustrated in Figure 2. A neuron that has fired at $\hat{t}$ will fire again at $t=$ $\hat{t}+T(\hat{t})$. Another neuron that has fired slightly later at $\hat{t}+\delta t^{\prime}$ fires its next spike at $t+\delta t$. If the input potential is constant between $t$ and $t+\delta t$, then 
$\delta t=\delta t^{\prime}$. If, however, $h$ increases between $t$ and $t+\delta t$, as is the case in Figure 2, then the firing time difference is reduced. The compression of firing time differences is directly related to an increase in the activity $A$. To see this, we note that all neurons that fire between $\hat{t}$ and $\hat{t}+\delta t^{\prime}$ must fire again between $t$ and $t+\delta t$. This is due to the fact that the network is homogeneous and the mapping $\hat{t} \rightarrow t=\hat{t}+T(\hat{t})$ is monotonous. If firing time differences are compressed, the population activity increases. For a $\mathrm{SRM}_{0}$ neuron with a kernel $\eta$ as in equation $2.7, \eta^{\prime}(s)>0$ holds for all $s>0$. An input with $h^{\prime}>0$ implies, because of equation 4.2, an increase of the activity: $h^{\prime}>0 \Longleftrightarrow A(t)>A\left(t-T_{b}\right)$.

For IF neurons we have a related result. To evaluate $C$, we use equation 2.10 and find

$$
C^{\mathrm{IF}}(t)=\tau \frac{h^{\prime}(t) e^{T_{b} / \tau}-h^{\prime}\left(t-T_{b}\right)}{\tilde{\eta}_{0}+h\left(t-T_{b}\right)+\tau h^{\prime}\left(t-T_{b}\right)} .
$$

The backward interval $T_{b}(t)$ is given implicitly by the threshold condition $\vartheta=h(t)-\left[\tilde{\eta}_{0}+h\left(t-T_{b}\right)\right] \exp \left(-T_{b} / \tau\right)$. It is shown in appendix B that the denominator on the right-hand side of equation 4.5 is always positive. Therefore, a compression of firing times and hence an increase of the activity occurs whenever the numerator of the equation is positive: $h^{\prime}(t)-h^{\prime}(t-$ $\left.T_{b}\right) \exp \left(-T_{b} / \tau\right)>0 \Longleftrightarrow A(t)>A\left(t-T_{b}\right)$. This is a rather general property of the noise-free IF dynamics that will be exploited in the following sections.

For general spike response neurons defined by equation $2.1, \partial_{t} h+\partial_{\hat{t}} h>0$ is a necessary condition for an increase of the activity, but it is not sufficient: $\partial_{t} h+\partial_{\hat{t}} h>0 \Longrightarrow A(t)>A\left(t-T_{b}\right)$. Details are discussed in appendix B.

4.2 Application to Locking. We will show in this subsection that the locking theorem developed in Gerstner et al. (1996) follows directly from the noise-free population equation 4.2. We consider a population that is already close to perfect synchrony and fires nearly regularly with period $T$. In order to keep the arguments transparent, let us assume that the population activity for times $t<T / 2$ can be approximated by a sequence of square pulses,

$$
A(t)=\sum_{n=-m}^{0} \frac{1}{2 \delta^{n}} \mathcal{H}\left(t-n T+\delta^{n}\right) \mathcal{H}\left(n T+\delta^{n}-t\right),
$$

where $m$ is some small integer, say, $m=3$, and $\mathcal{H}($.) denotes the Heaviside step function with $\mathcal{H}(s)=1$ for $s>0$ and $\mathcal{H}(s)=0$ for $s \leq 0$. The $\delta^{n}$ are assumed to be small, $\delta^{n} \ll T$, where $T$, is the period.

We want to check whether the "Ansatz" (see equation 4.6) is consistent with the noise-free population dynamics (see equation 4.2). More precisely, we ask, Is there some period $T$ and some sequence $\delta^{n}$ for $n=-m,-m+$ $1, \ldots, 0,1,2, \ldots$ so that the sequence of square pulses described by equation 4.6 continues for $t>T / 2$ ? We will determine $T$ and the sequence $\delta^{n}$ 
self-consistently. If we find $\delta^{n} \rightarrow 0$ for $n \rightarrow \infty$, then the square pulses contract to $\delta$-functions, and we say that a synchronous T-periodic oscillation is a stable solution of the population dynamics.

As a first step, we determine the potential $h_{\mathrm{PSP}}(t \mid \hat{t})$. Given $h_{\mathrm{PSP}}$, we can calculate the period $T$ from the threshold condition and also the derivatives $\partial_{t} h$ and $\partial_{\hat{t}} h$ needed in equation 4.2. To get $h_{\mathrm{PSP}}$, we put equation 4.6 in 3.3. We assume $\delta^{n} \ll T$ and integrate. To first order in $\delta^{n}$, we find

$$
h_{\mathrm{PSP}}(t \mid \hat{t})=\sum_{n=0}^{n_{\max }} J_{0} \epsilon(t-\hat{t}, t+n T)+\mathcal{O}\left[\left(\delta^{n}\right)^{2}\right]
$$

where $-\delta^{0} \leq \hat{t} \leq \delta^{0}$ is the last firing time of the neuron under consideration. The sum runs over all pulses back in the past. Since $\epsilon(t-\hat{t}, s)$ as a function of $s$ decays quickly for $s \gg T$, it is usually sufficient to keep only a finite number of terms (e.g., $n_{\max }=1$ or 2 ).

As the next step we determine the period $T$. To do so, we consider a neuron in the center of the square pulse that has fired its last spike at $\hat{t}=$ 0 . Since we consider noiseless neurons the relative order of firing of the neurons cannot change. To make the Ansatz, equation 4.6, consistent, the next spike of this neuron must therefore occur at $t=T$, in the center of the next square pulse. We use $\hat{t}=0$ in the threshold condition, equation 2.11, which yields

$$
T=\min \left\{t \mid \eta(t)+J_{0} \sum_{n=0}^{n_{\max }} \epsilon(t, t+n T)=\vartheta\right\} .
$$

If a synchronized solution exists, equation 4.8 defines the period.

In the population equation, 4.2 , we need the derivative of $h_{\mathrm{PSP}}$,

$$
\partial_{t} h+\partial_{\hat{t}} h=\left.J_{0} \sum_{n=0}^{n_{\max }} \frac{d}{d s} \epsilon(x, s)\right|_{x=T, s=n T} .
$$

According to equation 4.2, the new value of the activity at time $t=T$ is the old value multiplied by the factor in the square brackets. A necessary condition for an increase of the activity from one cycle to the next is that the derivative defined by the right-hand-side of equation 4.9 is positive, which is the essence of the locking theorem (Gerstner et al., 1996).

To illustrate the idea, let us study two specific cases: $\mathrm{SRM}_{0}$ neurons and IF neurons. For $\mathrm{SRM}_{0}$ neurons we have $\epsilon(x, s)=\epsilon_{0}(s)$, hence $\partial_{\hat{t}} h=0$ and $h_{\mathrm{PSP}}(t \mid \hat{t})=h(t)=J_{0} \sum_{n} \epsilon_{0}(t+n T)$. With the $\eta$ kernel defined by equation 2.7, we have $\eta^{\prime}(T)>0$ whatever $T$. Thus

$$
h^{\prime}(T)=J_{0} \sum_{n=1}^{n_{\max }+1} \epsilon_{0}^{\prime}(n T)>0 \quad \Longleftrightarrow \quad A(T)>A(0) .
$$




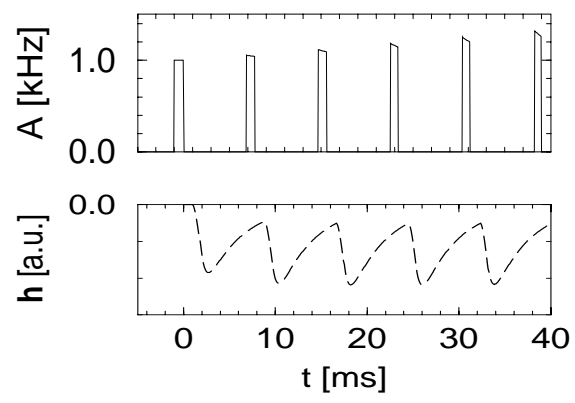

Figure 3: A sequence of activity pulses (top) contracts to $\delta$-pulses if firing always occurs when the input potential $h$ (dashed line, bottom) is rising. Numerical integration of the population equation, 3.7, for $\mathrm{SRM}_{0}$ neurons with inhibitory interaction $J=-0.1$ and kernel $\epsilon_{0}$ (see equation 2.5) with delay $\Delta^{\text {abs }}=2 \mathrm{~ms}$. There is no noise $(\sigma=0)$. The activity was initialized with a square pulse $A(t)=1 \mathrm{kHz}$ for $-1 \mathrm{~ms}<t<0$ and integrated with a step size of $0.05 \mathrm{~ms}$.

For IF neurons we could go through an analogous argument to show that equation 4.10 holds. Instead we may verify its validity directly from equation 4.5 with $h^{\prime}(t)=h^{\prime}\left(t-T_{b}\right)$ due to periodicity. Therefore the amplitude of the synchronous pulse grows only if $h^{\prime}(T)>0$.

The growth of amplitude corresponds to a compression of the width of the pulse. It can be shown that the corner neurons, which have fired at time $\pm \delta^{0}$, fire their next spike at $\delta^{1}=\delta^{0}[1+C]^{-1}$ with $C$ defined in equation 4.3. Thus the square pulse remains normalized, as it should be. By iteration of the argument for $t=n T$ with $n=2,3,4, \ldots$ we see that (for IF or $\mathrm{SRM}_{0}$ neurons) the sequence $\delta^{n}$ converges to zero, and the square pulses approach a Dirac $\delta$-pulse under the condition that $h^{\prime}(T)=\sum_{n} \epsilon_{0}^{\prime}(n T)>0$. In words, the $T$-periodic synchronized solution with $T$ given by equation 4.8 is stable if the input potential $h$ at the moment of firing is rising (Gerstner et al., 1996).

In order for the sequence of square pulses to be an exact solution of the population equation, we must require that the factor $C$ defined in equation 4.3 remains constant over the width of a pulse. The derivatives of equation 4.7 , however, do depend on $t$. As a consequence, the form of the pulse changes over time, as is visible in Figure 3. The activity as a function of time was obtained by a numerical integration of the population equation with a square pulse as the initial condition for a network of $S R M_{0}$ neurons coupled via equation 2.5 with weak inhibitory coupling $J=-0.1$ and delay $\Delta^{\mathrm{ax}}=2 \mathrm{~ms}$. For this set of parameters, $h^{\prime}(T)>0$ and locking is possible.

The framework of the population equation allows us also to extend the locking argument to noisy $\mathrm{SRM}_{0}$ neurons. At each cycle, the pulse of synchronous activity is compressed due to locking if $h^{\prime}(T)>0$. At the same time it is smeared out because of noise. For $\mathrm{SRM}_{0}$ neurons with gaussian noise 

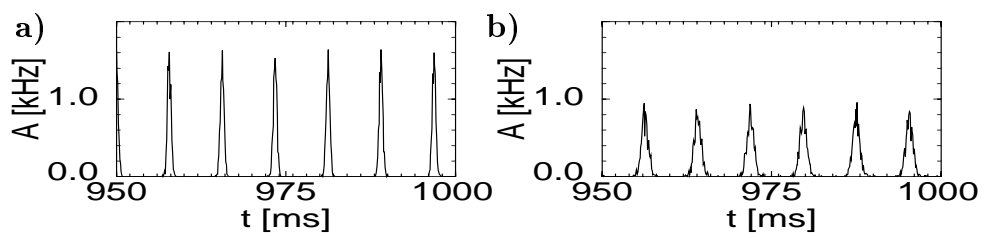

Figure 4: Synchronous activity in the presence of noise. Simulation of a population of 1000 neurons with inhibitory coupling $\left(J=-1, \Delta^{\text {ax }}=2 \mathrm{~ms}\right)$ and noise model B. (a) Low-noise level $(\sigma=0.25)$. (b) For larger noise $(\sigma=0.5)$, the periodic pulses become broader.

in the reset (noise model B), we may use equation 2.17 for the probability density in the population equation, 3.7,

$$
A(t)=\int_{-\infty}^{t} d \hat{t} \int_{-\infty}^{\infty} d r \delta[t-\hat{t}-T(\hat{t}, r)] \mathcal{G}_{\sigma}(r) A(\hat{t}),
$$

and search for periodic solutions. We insert $T(\hat{t}, r)=r+T_{0}(\hat{t}+r)$ where $T_{0}\left(t^{\prime}\right)$ is the forward interval of a noiseless neuron that has fired its last spike at $t^{\prime}$. The integration over $\hat{t}$ can be done and yields

$$
A(t)=\left[1+\frac{h^{\prime}}{\eta^{\prime}}\right] \int_{-\infty}^{\infty} d r \mathcal{G}_{\sigma}(r) A\left[t-T_{b}(t)-r\right]
$$

where $T_{b}$ is the backward interval. The factor $\left[1+\left(h^{\prime} / \eta^{\prime}\right)\right]$ arises due to the integration over the $\delta$-function just as in the noiseless case; see equations 4.2 and 4.4. The integral over $r$ leads to a broadening, the factor $\left[1+\left(h^{\prime} / \eta^{\prime}\right)\right]$ to a compression of the pulse.

As shown in appendix $C$, a limit cycle of equation 4.11 consisting of a periodic sequence of gaussian pulses exists if the noise amplitude $\sigma$ is small and $\left(h^{\prime} / \eta^{\prime}\right)>0$. The width of the activity pulses in the limit cycle is $d$ where $d=\sigma\left[2\left(h^{\prime} / \eta^{\prime}\right)+\left(h^{\prime} / \eta^{\prime}\right)^{2}\right]^{-1 / 2}$. As in the noise-free situation in equation 4.10, $h^{\prime}$ and $\eta^{\prime}$ have to be evaluated at T. A simulation of locking with noise is shown in Figure 4 . The network of $\mathrm{SRM}_{0}$ neurons has inhibitory connections $\left(J_{0}=-1\right)$ and is coupled via the response kernel $\epsilon_{0}$, equation 2.5, with a transmission delay of $\Delta^{\mathrm{ax}}=2 \mathrm{~ms}$. Doubling the noise level $\sigma$ leads to activity pulses with twice the width.

\section{Transients}

In this section we study the response of the population activity to a rapid change in the input. To keep the arguments as simple as possible, we con- 
sider an input that has a constant value $\mathcal{I}_{0}$ for $t<t_{0}$ and then changes abruptly to a new value $\mathcal{I}_{0}+\Delta \mathcal{I}$. Thus,

$$
\mathcal{I}^{\text {ext }}(t)= \begin{cases}\mathcal{I}_{0} & \text { for } t \leq t_{0} \\ \mathcal{I}_{0}+\Delta \mathcal{I} & \text { for } t>t_{0}\end{cases}
$$

For the sake of simplicity, we assume a population of independent IF or $\mathrm{SRM}_{0}$ neurons $\left(J_{0}=0\right)$. For $t \leq t_{0}$, all neurons receive a constant input potential $h_{0}=J^{\text {ext }} \mathcal{I}_{0}$ since $\int \tilde{\epsilon}_{0}(s) d s=1$. For $t>t_{0}$, the input potential, equation 2.4 , changes due to the additional current $\Delta \mathcal{I}$. Thus

$$
h(t)= \begin{cases}h_{0} & \text { for } t \leq t_{0} \\ h_{0}+J^{\text {ext }} \Delta \mathcal{I} \int_{0}^{t-t_{0}} \tilde{\epsilon}_{0}(s) d s & \text { for } t>t_{0}\end{cases}
$$

Given the input potential $h(t)$ and the last firing time $\hat{t}$, we can calculate for any given neuron its momentary membrane potential $u(t)$-but what is the time course of the population activity?

In order to analyze the situation, let us suppose that for $t<t_{0}$, the network is in a state of incoherent firing. In other words, all neurons fire at the same mean firing rate, but their spikes are not synchronized. Rather, the firing times are maximally spread out over time. Such a state has been called the splay phase (Chow, 1998). In the limit of $N \rightarrow \infty$, the population activity is then a constant $A(t)=A_{0}$. In section 7 we will study the conditions under which a state of incoherent firing can be a stable state of the system. Here we just assume that we can set the parameters so that the network fires asynchronously and with constant activity.

5.1 Transients in a Noise-Free Network. In the noiseless case, neurons that receive a constant input $\mathcal{I}_{0}$ fire regularly with some period $T_{0}$. For $t<t_{0}$, the mean activity is simply $A_{0}=1 / T_{0}$. The reason is that, for a constant activity, averaging over time and averaging over the population must be the same.

We now apply the equation of the noise-free population dynamics: equation 4.2. To do so, we have to calculate the factor $C(t)$ defined in equation 4.3. For IF neurons, $C$ is given by equation 4.5 and for SRM neurons by equation 4.4. In both cases we need the derivative of equation 5.2:

$$
h^{\prime}(t)=\left\{\begin{array}{cc}
0 & \text { for } t \leq t_{0} \\
J^{\text {ext }} \Delta \mathcal{I} \tilde{\epsilon}_{0}\left(t-t_{0}\right) & \text { for } t>t_{0}
\end{array} .\right.
$$

For $t<t_{0}$, we have $h^{\prime}=0$ and thus, from equation 4.2, $A(t)=A\left(t-T_{0}\right)$, as it should be for a constant activity $A_{0}$. Let us now consider a neuron that has fired exactly at $t_{0}$. Its next spike occurs at $t_{0}+T$, where $T$ is given by 
the threshold condition $u_{i}\left(t_{0}+T\right)=\vartheta$. If the change $\Delta \mathcal{I}$ at $t_{0}$ is small, we expect that the interspike interval $T$ changes only slightly. In order to keep the arguments transparent, we make a zero-order approximation and set $\exp (-T / \tau) \approx \exp \left(-T_{0} / \tau\right)$, hence $\eta^{\prime}(T) \approx \eta^{\prime}\left(T_{0}\right)$. The population equation, 4.2 , then yields

$$
A(t)=\left[1+a \tilde{\epsilon}_{0}\left(t-t_{0}\right)\right] A_{0} \quad \text { for } \quad t_{0}<t<t_{0}+T,
$$

with a constant $a=J^{\mathrm{ext}} \Delta \mathcal{I} / \eta^{\prime}\left(T_{0}\right)$ for $\mathrm{SRM}_{0}$ neurons and $a=J^{\text {ext }} \Delta \mathcal{I}$ / $\left[\eta^{\prime}\left(T_{0}\right)+h_{0} \exp \left(-T_{0} / \tau\right)\right]$ for IF neurons. Thus, the time course of the initial transient reflects the time course $\tilde{\epsilon}_{0}$ of the postsynaptic potential caused by external input.

So far, the results are general. We now specify the response kernel $\tilde{\epsilon}_{0}$. With equation 2.6, the response of the input potential to the step current is $h(t)=h_{0}+J^{\text {ext }} \Delta \mathcal{I}\left[1-\exp \left[-\left(t-t_{0}\right) / \tau\right]\right]$ for $t>t_{0}$. Thus, the potential has the characteristics of a low-pass filter. The population activity, however, reacts instantaneously to the step current. We put equation 2.6 in 5.4 and find

$$
A(t)=A_{0}+\Delta A \frac{1}{\tau} \exp \left(-\frac{t-t_{0}}{\tau}\right) \mathcal{H}\left(t-t_{0}\right) \quad \text { for } t_{0}<t<t_{0}+T,
$$

where $\Delta A=A_{0} a$ and $\mathcal{H}($.$) is the Heaviside step function. Thus there is an$ immediate response at $t=t_{0}$. The simulation in Figure 5 clearly exhibits the rapid initial response of the population. It is also confirmed by a numerical integration of the noise-free population dynamics, equation 4.2. As an aside we note that a dynamic rate model of the form of equation 1.2 defined by a differential equation with time constant $\tau$ would not be able to capture the initial phase of the transient.

The discussion up to now has focused on the initial phase of the transient. For $t>t_{0}+T$ every neuron has fired once, and the activity on the right-hand side of equation 4.2 can no longer be considered constant. What happens? Let us treat the IF model first. With the response kernel $\tilde{\epsilon}_{0}$ defined in equation 2.6, we find for $t>t_{0}+T$ the relation $h^{\prime}(t-T)=h^{\prime}(t) \exp (T / \tau)$. Hence $C^{\mathrm{IF}}$ defined in equation 4.5 vanishes for $t>t_{0}+T$. Therefore $A(t)=A(t-T)$, where $T$ is the new backward interval consistent with the constant stimulation current $I_{0}+\Delta \mathcal{I}$. Thus the population activity is in a new periodic state as soon as every neuron has fired once. This is what we should expect since neurons are independent, and as soon as each has been reset once after $t_{0}$, they all feel the same constant input current.

The picture is different for $\mathrm{SRM}_{0}$ neurons. Here the new periodic state is reached only asymptotically (see Figure 5). The sequence of peaks in the activity that occur at times $t_{k} \approx t_{0}+k T_{0}$ is given by the iteration $A\left(t_{k+1}\right)=$ $A\left(t_{k}\right)\left[1+h^{\prime}\left(t_{k+1}\right) / \eta^{\prime}\left(T_{0}\right)\right]$. Since $h^{\prime}(t) \propto \exp \left[-\left(t-t_{0}\right) / \tau\right]$, the new limit cycle is approached asymptotically with time constant $\tau$. For $\mathrm{SRM}_{0}$ neurons, the 

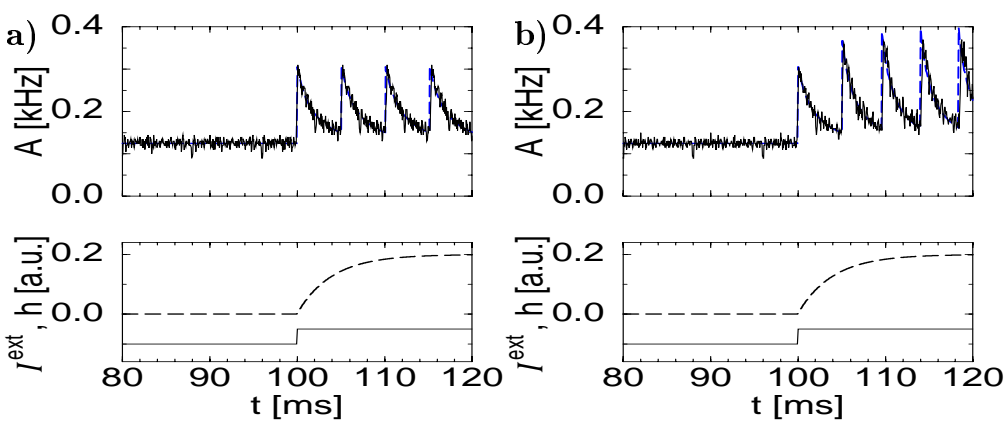

Figure 5: (Top) Response of the population activity to a step current for low noise $(\sigma=0.01 \mathrm{~ms})$. Solid line: Simulation of a population of 1000 neurons. Dashed line: Numerical integration of the population equation, 3.7 (a) IF neurons. (b) $\mathrm{SRM}_{0}$ neurons. (Bottom) Step current input $I^{\text {ext }}$ (solid line) and input potential $h(t)$ (dashed line). Parameters: Time constant $\tau=4 \mathrm{~ms}$ with response kernel $\tilde{\epsilon}_{0}$ defined in equation 2.6; input step of 0.05 units at $t=100 \mathrm{~ms}$. The threshold $\vartheta$ was chosen so that the mean interval before the step is $8 \mathrm{~ms}$, corresponding to a mean activity of $0.125 \mathrm{kHz}$. There are no interactions, $J=0$; integration time step, $0.05 \mathrm{~ms}$. The result of the simulation has been smoothed with a running average over $0.2 \mathrm{~ms}$.

sequence of activity peaks therefore follows roughly the same exponential time course as the input potential $h$.

5.2 Transients with Noise. So far we have considered noiseless neurons. In the presence of noise, subsequent pulses get smaller and broader, and the network approaches a new incoherent state (cf. Figure 6a). If, in
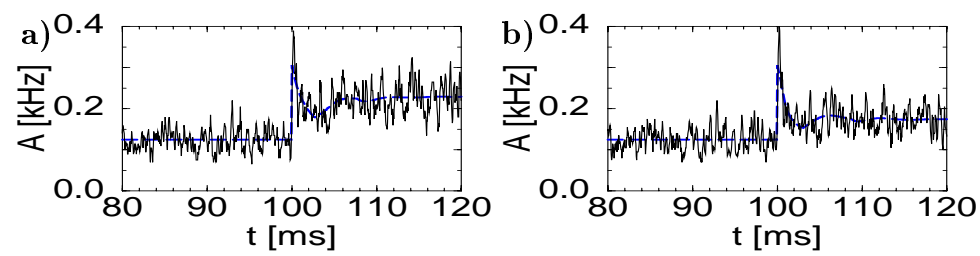

Figure 6: Transients for $\mathrm{SRM}_{0}$ neurons with noise model B. (a) Same as in Figure $5 \mathrm{~b}$, but at a noise level of $\sigma=2$ and $J=0$. (b) Noisy network $(\sigma=2)$ with inhibitory interactions defined by equation 2.5 with $\Delta^{\mathrm{ax}}=0.5 \mathrm{~ms}$ and $J=-2$. In both cases the results of a simulation of $1000 \mathrm{SRM}_{0}$ neurons (solid line) are compared with a numerical integration (dashed line) of equation 3.7. All other parameters are as in Figure 5. 
addition, there is also some interaction $J_{0} \neq 0$ between the neurons, then the value of the new stationary state is shifted. A particularly interesting state is inhibitory interaction $J_{0}<0$. In this case the new value of the stationary activity for $t>t_{0}$ is only slightly higher than that for $t<t_{0}$. For noise model $\mathrm{B}$, the fast transient with a sharp first peak is clearly visible and marks the moment of input switching (see Figure 6b). Fast switching has previously been seen in heterogeneous networks with balanced excitation and inhibition (Tsodyks \& Sejnowski, 1995; van Vreeswijk \& Sompolinsky, 1996) and also discussed in the context of associative memory: Treves, 1992; Treves, Rolls, \& Simmen, 1997). Here it is demonstrated and analyzed for homogeneous networks.

For a preliminary analysis of the noisy case, we take $\mathrm{SRM}_{0}$ neurons with noise model B and start from equation 4.12. To simplify the expression, we write $A(t)=A_{0}+\Delta A(t)$ and expand equation 4.12 to first order in $\Delta A$. The result is

$$
\Delta A(t)=\int_{-\infty}^{\infty} \mathcal{G}_{\sigma}(r) \Delta A\left(t-T_{0}-r\right) d r+\frac{h^{\prime}(t)}{\eta^{\prime}\left(T_{0}\right)} A_{0} .
$$

The result for IF neurons with noise model B is analogous to equation 5.6 and is derived in appendix D.

The first term in equation 5.6 accounts for changes that are due to previous variations of the activity. During the initial phase of the transient (for $t_{0}<$ $\left.t<t_{0}+T_{0}-\sigma\right)$, it plays no role and can be neglected. Thus the initial phase is determined by the second term. As in the noiseless case, the initial transient is proportional to the derivative of $h$. After this initial phase, the convolution with the gaussian (the first term on the right-hand side of equation 5.6) comes into play and leads to a rapid smoothing (see Figure 6). The initial transient, however, is sharp.

We may wonder whether we can understand fast switching intuitively. Before the abrupt change, the input was stationary and the population in a state of incoherent firing, defined as a state where neuronal firing times are spread out maximally. Thus some of the neurons fire, others are in the refractory period, and again others approach the threshold. There is always a group of neuron whose potential is just below threshold. An increase in the input causes those neurons to fire immediately, and this accounts for the strong population response during the initial phase of the transient.

The above consideration also holds for noise model C (noisy integration) in the limit of low noise and suprathreshold inputs. In order to understand why the derivative of $h$ comes into play, consider a finite step in the input potential $h_{1}(t)=\Delta h \mathcal{H}\left(t-t_{0}\right)$. All neurons $i$ that are hovering below threshold so that their potential $u_{i}\left(t_{0}\right)$ is between $\vartheta-\Delta h$ and $\vartheta$ will be put above threshold and fire synchronously at $t_{0}$. Thus, a step in the potential causes a $\delta$-pulse in the activity $\Delta A(t) \propto \delta\left(t-t_{0}\right) \propto h^{\prime}\left(t_{0}\right)$. In Figure 7a we have used a current step (see equation 5.1), the same step current as in Figure 5. The 

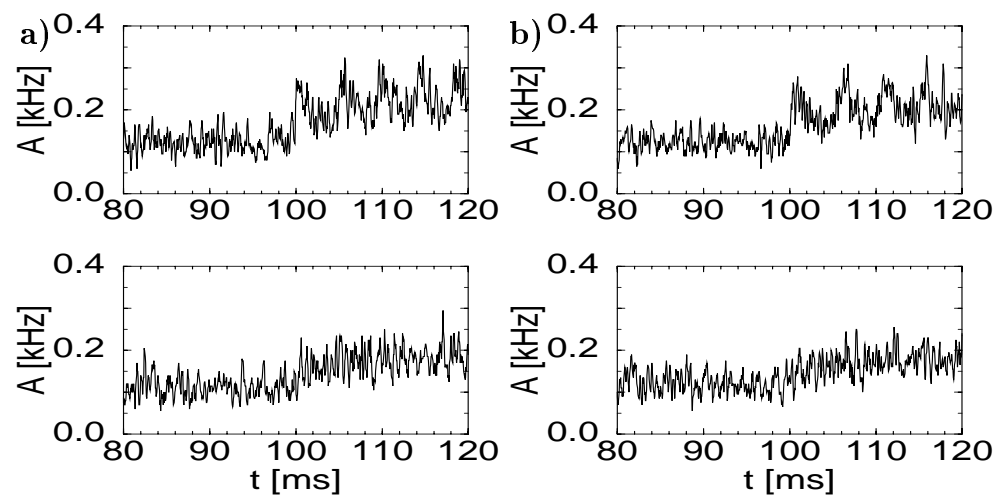

Figure 7: Response of a network of 1000 neurons without interaction $(J=0)$ to step current input as in Figure 5, but for (a) noise model C (noisy integration) and (b) noise model A (noisy threshold). For low noise, the transition is sharp (top row), whereas for high noise rather smooth (bottom). In all cases, threshold and noise level were adjusted so that the mean interval before the current step is $8 \mathrm{~ms}$ so as to allow a comparison with Figure 5. (Top) Noise level chosen so that the variance of the interval distribution is $1 \mathrm{~ms}$. (Bottom) Variance about $4 \mathrm{~ms}$.

response at low noise (top) has roughly the form $\Delta A(t) \propto h^{\prime}(t) \propto \tilde{\epsilon}_{0}\left(t-t_{0}\right)$, as expected. The rapid transient is slightly less pronounced than for noise model B, but nevertheless clearly visible; compare Figures 6 and 7. As the amplitude of the noise grows, the transient becomes less sharp. Thus there is a transition from a regime where the transient is proportional to $h^{\prime}$ (see Figure 7a, top) to another regime where the transient is proportional to $h$ (see Figure 7a, bottom). What are the reasons for the change of behavior?

The above simple argument based on a potential step $\Delta h>0$ holds only for a finite step size, which is at least of the order of the noise amplitude $\sqrt{D}$. In noise model $C$, the threshold acts as an absorbing boundary. Therefore the density of neurons with potential $u_{i}$ vanishes for $u_{i} \rightarrow \vartheta$. Thence, for $\Delta h \rightarrow 0$, the proportion of neurons that are instantaneously put across threshold is 0 . In a stationary state, the boundary layer with low density is of the order $\sqrt{D}$ (Brunel \& Hakim, 1999). A potential step $\Delta h>\sqrt{D}$ puts a significant proportion of neurons above threshold and leads to a $\delta$-pulse in the activity. Thus the result that the response is proportional to the derivative of the potential is essentially valid in the low-noise regime.

On the other hand, noise model $\mathrm{C}$ can also be used in the regime of large-noise and subthreshold input. In the high-noise regime, a step in the potential raises the instantaneous rate of the neurons but does not force them to fire immediately. The response to a current step is therefore smooth and 
follows the potential $h(t)$ (see Figure 7a, bottom). A comparison of Figures 7a and $7 \mathrm{~b}$ shows that noise model A exhibits a similar transition from sharp to smooth responses with increasing noise level. It can be shown that at least in the subthreshold regime, noise model $\mathrm{C}$ can be well approximated by noise model A (Plesser \& Gerstner, 1999; see also Collins, Carson, Capela, \& Imhoff, 1996). Analytical arguments for transients with noise model A are presented below.

5.3 Theory of Transients with Noise. To formalize the above ideas on transient responses in the presence of noise, we start from the population equation, 3.7, and write it in the form

$$
0=\frac{d}{d t} \int_{-\infty}^{t} S_{h}(t \mid \hat{t}) A(\hat{t}) d \hat{t}
$$

Equation 3.7 is the derivative of the conservation law, equation 3.6, and this is made explicit by our notation in equation 5.7 .

We consider a small perturbation of the stationary state $A(t)=A_{0}+\Delta A(t)$ and $h_{\mathrm{PSP}}(t \mid \hat{t})=h_{0}(t \mid \hat{t})+h_{1}(t \mid \hat{t})$ and expand equation 5.8 to linear order in $\triangle A$ or $h_{1}$ :

$$
\begin{aligned}
0= & \frac{d}{d t} \int_{-\infty}^{t} S_{0}(t-\hat{t}) \Delta A(\hat{t}) d \hat{t} \\
& +A_{0} \frac{d}{d t}\left\{\left.\int_{-\infty}^{t} d t_{1} \int_{-\infty}^{t} d \hat{t} h_{1}\left(t_{1} \mid \hat{t}\right) \frac{\partial S_{h}(t \mid \hat{t})}{\partial h_{1}\left(t_{1} \mid \hat{t}\right)}\right|_{h_{1}=0}\right\} .
\end{aligned}
$$

We have used the notation $S_{0}(t-\hat{t})=S_{h_{0}}(t \mid \hat{t})$ for the survivor function of the asynchronous firing state. For the derivative in the first term in equation 5.8, we use $\frac{d}{d t} S_{0}(t-\hat{t})=-P_{h_{0}}(t \mid \hat{t})$ and $S_{0}(0)=1$.

To make some progress in the treatment of the second term in equation 5.8, it is helpful to take either the $\mathrm{SRM}_{0}$ or the IF model. For $\mathrm{SRM}_{0}$ neurons, we may drop the $\hat{t}$ dependence of the potential and set $h_{1}\left(t_{1} \mid \hat{t}\right)=h_{1}\left(t_{1}\right)$, which allows us to pull the $h_{1}\left(t_{1}\right)$ in front of the integral over $\hat{t}$ and write equation 5.8 in the form

$$
\Delta A(t)=\int_{-\infty}^{t} P_{h_{0}}(t \mid \hat{t}) \Delta A(\hat{t}) d \hat{t}+A_{0} \frac{d}{d t}\left\{\int_{0}^{\infty} \mathcal{L}(x) h_{1}(t-x) d x\right\},
$$

with a filter $\mathcal{L}=\mathcal{L}^{\mathrm{SRM}}$ defined in the top row of Table 1 . For IF neurons we set $h_{1}(t \mid \hat{t})=h_{1}(t)-h_{1}(\hat{t}) \exp [-(t-\hat{t}) / \tau]$. After some rearrangements of the terms, equation 5.8 becomes identical to 5.9 with a filter $\mathcal{L}=\mathcal{L}^{\mathrm{IF}}$ (see Table 1).

The first term on the right-hand side of equation 5.9 is of the same form as the population equation 3.7 and describes how perturbations $\Delta A(\hat{t})$ in 
Table 1: Filter $\mathcal{L}(x)$ for Integrate-and-Fire or $\mathrm{SRM}_{0}$ neurons (Upper index IF or SRM, respectively).

\begin{tabular}{|c|c|}
\hline Def & $\begin{aligned} \mathcal{L}^{\mathrm{SRM}}(x) & =-\int_{x}^{\infty} d \xi \frac{\partial S(\xi \mid 0)}{\partial h_{1}(\xi-x)} \\
\mathcal{L}^{\mathrm{IF}}(x) & =\mathcal{L}^{\mathrm{SRM}}(x)+\int_{0}^{x} d \xi e^{-\xi / \tau} \frac{\partial S(x \mid 0)}{\partial h_{1}(\xi)}\end{aligned}$ \\
\hline A & $\begin{aligned} \mathcal{L}_{A}^{\mathrm{SRM}}(x) & =\int_{x}^{\infty} d \xi f^{\prime}[u(\xi-x)] S_{0}(\xi) \\
\mathcal{L}_{A}^{\mathrm{IF}}(x) & =\mathcal{L}_{A}^{\mathrm{SRM}}(x)-S_{0}(x) \int_{0}^{x} d \xi e^{-\xi / \tau} f^{\prime}[u(\xi)]\end{aligned}$ \\
\hline B & $\begin{aligned} \mathcal{L}_{B}^{\mathrm{SRM}}(x) & =\delta(x) / \eta^{\prime} \\
\mathcal{L}_{B}^{\mathrm{IF}}(x) & =\left[\delta(x)-\mathcal{G}_{\hat{\sigma}}\left(x-T_{0}\right) e^{-T_{0} / \tau}\right] / u^{\prime}\end{aligned}$ \\
\hline
\end{tabular}

Notes: $f^{\prime}=d f / d u$ is the derivative of the escape function $f(u) ; S_{0}(s)=S_{h_{0}}(s \mid 0)$ is the survivor function in the incoherent state. For noise model $\mathrm{B}$, the width of the gaussian is $\hat{\sigma}=\sigma T_{1}=\sigma \tilde{\eta}_{0} /\left(\tilde{\eta}_{0}+h_{0}\right)$ and the derivatives are $\eta^{\prime}=d \eta /\left.d s\right|_{s=T_{0}}$ and $u^{\prime}=\eta^{\prime}+\tau^{-1} h_{0} \exp \left(-T_{0} / \tau\right)$. In B, the approximation $\exp (\hat{\sigma} / \tau) \approx 1$ has been used.

the past influence the current activity $\Delta A(t)$. The second term gives an additional contribution, which is proportional to the derivative of a filtered version of the potential. As we have seen, for $\mathrm{SRM}_{0}$ neurons with noise model B, we find $\mathcal{L}(x)=\mathcal{L}_{B}^{\mathrm{SRM}}(x)=\left(1 / \eta^{\prime}\right) \delta(x)$. Thus for noise model B, the filter is a $\delta$-function, and the second term in equation 5.9 is proportional to $h^{\prime}$.

On the other hand, it is shown in appendix $\mathrm{E}$ that for noise model $\mathrm{A}, \mathcal{L}$ can often be approximated by a low-pass filter,

$$
\mathcal{L}_{A}^{\mathrm{SRM}}(x)=a \rho e^{-\rho x} \mathcal{H}(x)
$$

where $a$ is a constant and $\rho$ is a measure of the noise. (The result for IF neurons is given in appendix E.) The noise-free threshold process can be retrieved from equation 5.10 for $\rho \rightarrow \infty$. In this limit $\mathcal{L}_{A}^{\mathrm{SRM}}(x)=a \delta(x)$, and the initial transient is proportional to $h^{\prime}$, as discussed above. For small $\rho$, however, the behavior is different. We use equation 5.10 and rewrite the last term in equation 5.9 in the form

$$
\frac{d}{d t} \int_{0}^{\infty} \mathcal{L}_{A}^{\mathrm{SRM}}(x) h_{1}(t-x) d x=a \rho\left[h_{1}(t)-\bar{h}_{1}(t)\right],
$$

where $\bar{h}_{1}(t)=\int_{0}^{\infty} \rho \exp (-\rho x)$ is a running average. Thus the activity responds to the temporal contrast $h_{1}(t)-\bar{h}_{1}(t)$. At high-noise levels, $\rho$ is small. During the initial phase of the transient $\left(t-t_{0}<\rho^{-1}\right)$, we may set $\bar{h}_{1}(t)=0$. Thus, we find for noise model $\mathrm{A}$ in the large-noise limit $\Delta A(t) \propto h(t)$. This is 
exactly the result that would be expected for a simple rate model. For a simulation of noise model A, see Figure $7 \mathrm{~b}$. The generalization of equation 5.11 to IF neurons is straightforward (cf. appendix E).

In all cases, the initial phase of the transient is proportional to a linearly filtered version of the potential, $\Delta A \propto \frac{d}{d t}\{\mathcal{L} * h\}$. In the limit of low noise, the choice of noise model is irrelevant; the transient response is proportional to the derivative of the potential, $\Delta A \propto h^{\prime}$. If the level of noise is increased, noise model $B$ retains its sharp transients, whereas $A$ and $C$ turn to a different regime where the transients follow $h$ rather than $h^{\prime}$. The transition from one regime to the other is most easily studied for noise model $\mathrm{A}$. The filter $\mathcal{L}$ is essentially a low-pass filter. The time constant of the filter increases with the noise level.

The results have an interesting relation to experimental input-output measurements on motoneurons (Fetz and Gustafsson, 1983; Poliakov, Powers, \& Binder, 1997). In the low-noise regime (where the type of noise model is irrelevant) the response to a synaptic input current pulse is proportional to the derivative of the postsynaptic potential (Fetz \& Gustafsson, 1983), as predicted by earlier theories (Knox, 1974). On the other hand, we have seen that for noise models $A$ and $C$, the behavior changes at higher noise levels and the response follows $h$ rather than $h^{\prime}$. At intermediate noise levels, the response to a synpatic input is therefore proportional to some mixture between the postsynaptic potential and its derivative (Fetz \& Gustafsson, 1983; Poliakov et al., 1997).

For noise model $\mathrm{B}$, the initial response to a current step is $\Delta A \propto \tilde{\epsilon}_{0}\left(t-t_{0}\right)$, independent of the noise level. Note that if the rise time of the postsynaptic potential $\tilde{\epsilon}_{0}$ is slow (as it is the case for slow synaptic channels), the transient will be slow as well. In other words, the response time is limited by the time constant of the synpatic current rather than by the membrane time constant. In the following section, this statement will be made more precise.

\section{Signal Transmission}

Our considerations regarding step current input can be generalized to arbitrary input current $I^{\text {ext }}(t)$. We study a population of independent IF or $\mathrm{SRM}_{0}$ neurons. For each neuron, the membrane potential can be calculated from the input potential $h(t)=J^{\text {ext }} \int_{0}^{\infty} \tilde{\epsilon}_{0}(s) I^{\text {ext }}(t-s) d s$ and the last firing time $\hat{t}$ (cf. equations 2.1 and 2.10). We assume that the population is close to a state of asynchronous firing: $A(t)=A_{0}+\Delta A(t)$. The linear response of the population to the change in the potential is given by equation 5.9 . The Fourier transform is

$$
\hat{A}(\omega)=i \omega \frac{J^{\mathrm{ext}} A_{0} \hat{\mathcal{L}}(\omega) \hat{\tilde{\epsilon}}(\omega)}{1-\hat{P}(\omega)} \hat{\mathcal{I}}(\omega)
$$



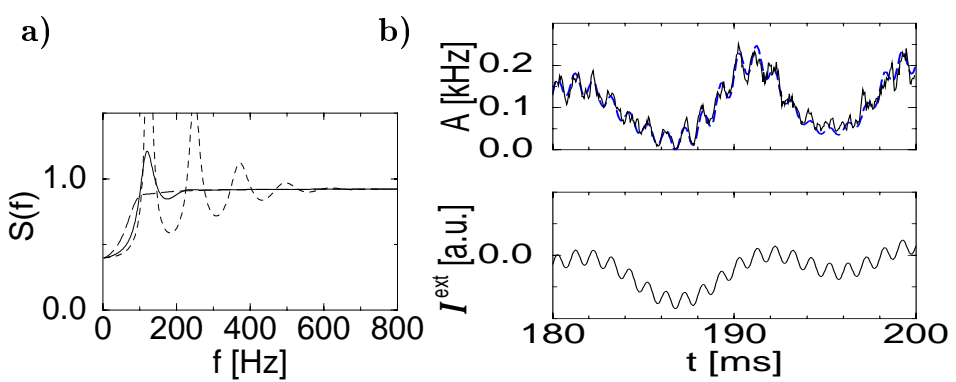

Figure 8: (a) Signal gain $S(f)=|\hat{A}(2 \pi f) / \hat{\mathcal{I}}(2 \pi f)|$ as a function of the frequency $f$ for IF neurons with time constant $\tau=4 \mathrm{~ms}$ and noise model $\mathrm{B}$ and a mean interspike interval of $8 \mathrm{~ms}$. Resonances are at the multiples of the single-neuron frequency of $125 \mathrm{~Hz}$. Noise level $\sigma=4 \mathrm{~ms}$ (long-dashed line); $\sigma=2 \mathrm{~ms}$ (solid line); $\sigma=0.75 \mathrm{~ms}$ (short-dashed); $J^{\text {ext }}=1$. (b) Response of the population activity (top) of $\mathrm{SRM}_{0}$ neurons with noise model B to a time-dependent current (bottom). The current is a superposition of 4 sine waves at 9, 47, 111, and $1000 \mathrm{~Hz}$. The simulation of a population of 4000 neurons (solid line, top) is compared with the numerical integration (dashed line) of the population equation, 3.7. Note that even the $1 \mathrm{kHz}$ component of the signal is well transmitted. Parameters: Response function $\tilde{\epsilon}_{0}$ (see equation 2.6) with time constant $\tau=4 \mathrm{~ms}$. Threshold is $\vartheta=-0.135$ so that the mean activity is $A=125 \mathrm{~Hz}$; noise $\sigma=2 \mathrm{~ms} ; J_{0}=0$.

Hats denote transformed quantities; $\hat{\tilde{\epsilon}}(\omega)=[1+i \omega \tau]^{-1}$ is the Fourier transform of the kernel $\tilde{\epsilon}_{0}$ defined in equation $2.6 \hat{P}(\omega)$ is the Fourier transform of the interval distribution, $I(\omega)$ is the Fourier transform of the current $I^{\text {ext }}$, and $\hat{\mathcal{L}}(\omega)$ is the transform of the linear filter $\mathcal{L}$.

To be specific, we consider IF neurons with noise model B. For gaussian reset we find $\hat{P}(\omega)=\exp \left\{-\frac{1}{2} \hat{\sigma}^{2} \omega^{2}-i \omega T_{0}\right\}$. The filter $\mathcal{L}$ may be read off Table 1 and gives $\hat{\mathcal{L}}(\omega)=\left\{1-\exp \left[-\frac{1}{2} \hat{\sigma}^{2} \omega^{2}-i \omega T_{0}-T_{0} / \tau\right]\right\} / u^{\prime}$ where $u^{\prime}$ is to be evaluated at $T_{0}$. In Figure 8a we have plotted the gain $S=|\hat{A}(\omega) / \hat{\mathcal{I}}(\omega)|$ as a function of the frequency $f=\omega /(2 \pi)$. For a medium noise level of $\sigma=2 \mathrm{~ms}$, the signal gain has a single resonance at $f=1 / T_{0}=125 \mathrm{~Hz}$. For lower noise, further resonances at multiples of $125 \mathrm{~Hz}$ appear. For a variant of noise model B, a result closely related to equation 6.1 has been derived by Knight (1972a). The result for $\mathrm{SRM}_{0}$ neurons with noise model B is obtained by neglecting a term $\propto \exp \left(-T_{0} / \tau\right)$.

Independent of the noise level, we obtain for IF neurons for $\omega \rightarrow 0$ the result $S(0)=J_{\text {ext }} A_{0}\left[1-\exp \left(-T_{0} / \tau\right)\right] /\left(u^{\prime} T_{0}\right)$. Most interesting is the behavior in the high-frequency limit. For $\omega \rightarrow \infty$ we find $S \rightarrow J^{\text {ext }} A_{0} /\left(u^{\prime} \tau\right)$, hence

$$
\frac{S(\infty)}{S(0)}=\frac{T_{0}}{\tau}\left[1-e^{-T_{0} / \tau}\right]^{-1} .
$$


We emphasize that the high-frequency components of the current are not attenuated by the population activity despite the integration on the level of the individual neurons. The reason is that the threshold process acts as a differentiator and reverses the low-pass filtering of the integration. In fact, equation 6.2 shows that high frequencies can be transmitted more effectively than low frequencies. The good transmission characteristics of spiking neurons at high frequencies have been studied in (Knight, 1972a) and confirmed experimentally (Knight, 1972b).

In biology there is, of course, also a time constant of synaptic channels that leads to a frequency cutoff for the input current that may enter the cell. In this sense, it is the time constant of the synaptic current that determines the cutoff frequency of the population. The membrane time constant is of minor influence. Treves (1993) has reached a similar conclusion.

So far we have discussed results of the linearized theory (see equations 5.6 and 6.1). The behavior of the full nonlinear system is shown in Figure $8 \mathrm{~b}$. A population of unconnected $\mathrm{SRM}_{0}$ neurons is stimulated by a time-dependent input current that was generated as a superposition of four sinusoidal components with frequencies at 9, 47, 111, and $1000 \mathrm{~Hz}$, which have been chosen arbitrarily. The activity equation, 3.7, has been integrated with time steps of $0.05 \mathrm{~ms}$, and the results are compared with those of a simulation of a population of 4000 neurons. The $1 \mathrm{kHz}$ component of the signal $\mathcal{I}(t)$ is clearly reflected in the population $A(t)$. Theory and simulation are in excellent agreement.

We have seen that noise model B was rather exceptional in that the transient remained sharp even in the limit of high noise. To study the relevance of the noise model, we return to equation 6.1. The signal gain $S(\omega)=|\hat{A}(\omega) / \hat{I}(\omega)|$ is proportional to $\hat{\mathcal{L}}(\omega)$. If the filter $\mathcal{L}(x)$ is broad, the Fourier transform will fall off to zero at high frequencies, and so does the signal gain $S(\omega)$. In Figure 9 we have plotted the filter $\mathcal{L}_{A}^{\mathrm{IF}}$ and the signal gain $S$ for IF neurons with noise model A at different noise levels. At low noise, the result for noise model A is similar to that of noise model B (compare Figures $9 b$ and $8 a$ ) except for a drop of the gain at high frequencies. Increasing the noise level, however, lowers the signal gain of the system. For high noise (the long-dashed line in Figure 9) the signal gain at $1000 \mathrm{~Hz}$ is 10 times lower than the gain at zero frequency. Note that for noise model A, the gain at zero frequency changes with the level of noise.

\section{Incoherent Firing}

In the preceding sections it was shown that a population of neurons may react rapidly to changes in the input if the network is in a state of incoherent firing. For a constant input current, incoherent firing may be defined as a macroscopic firing state with constant activity $A(t)=A_{0}$. In other words, incoherent firing corresponds to a fixed point of the population dynamics. 

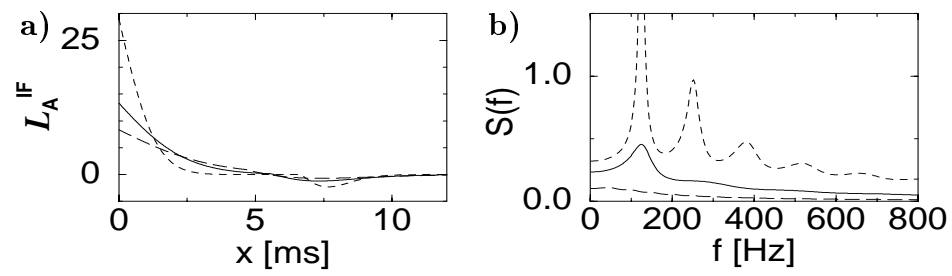

Figure 9: The filter $\mathcal{L}_{A}^{\mathrm{IF}}(x)$ (a) and the signal gain (b) for IF neurons with noise model A and escape rate $\rho=\rho_{0}[u-\vartheta] \mathcal{H}(u-\vartheta)$. For low noise (short-dashed line, $\rho_{0}=20$ which corresponds to a variance of the interval distribution of $\sigma=0.75 \mathrm{~ms}$ ), the filter $\mathcal{L}_{A}^{\mathrm{IF}}$ has a small width. For high noise (long-dashed line; $\rho_{0}=2.5$; variance $\sigma=4 \mathrm{~ms}$ ), the filter $\mathcal{L}_{A}^{\mathrm{IF}}$ is broad. Solid line: $\rho_{0}=5.5$, which corresponds to an interval distribution with $\sigma=2 \mathrm{~ms}$. The value of the threshold has been adjusted so that the mean interval is always $8 \mathrm{~ms}$. At low noise, the signal gain shows several resonances at the single-neuron frequency of $125 \mathrm{~Hz}$. For high frequencies, the signal gain declines due to the low-pass characteristics of the filter $\mathcal{L}_{A}^{\mathrm{IF}}$. In (a) the slightly negative valley around $T_{0}=8 \mathrm{~ms}$ is typical for IF neurons and would be absent for $\mathrm{SRM}_{0}$ neurons.

In this section we study the existence and stability of incoherent firing states.

7.1 Determination of the Activity. We search for a fixed point $A(t)=A_{0}$ of the population dynamics. Given constant $A_{0}$ and constant external input $\mathcal{I}_{0}$, the input potential of IF or $\mathrm{SRM}_{0}$ neurons is also constant,

$$
h(t)=h_{0}=J_{0} A_{0}+J^{\mathrm{ext}} \mathcal{I}_{0},
$$

where we have used the normalization $\int \epsilon_{0}(s) d s=1=\int \tilde{\epsilon}_{0}(s) d s$. In a noisefree situation, neurons driven by $h_{0}$ fire regularly with a period $T_{0}$, which may be determined directly from the threshold condition. The population activity is then $A_{0}=1 / T_{0}$. In the noisy case, the activity is $A_{0}=1 /\langle T\rangle$, where $\langle T\rangle=\int_{0}^{\infty} s P_{h_{0}}(\hat{t}+s \mid \hat{t}) d s$ is the mean interval length (Gerstner, 1995). The statement follows from the normalization, equation 3.6. For constant input potential $h_{0}$, the survivor function and the interval distribution cannot depend explicitly on the absolute time, but only on the time difference $t-\hat{t}$. Hence we may set $S_{0}(s)=S_{h_{0}}(\hat{t}+s \mid \hat{t})$ and $P_{0}(s)=P_{h_{0}}(\hat{t}+s \mid \hat{t})$. The normalization reduces to

$$
1=A_{0} \int_{0}^{\infty} s_{0}(s) d s=A_{0} \int_{0}^{\infty} s P_{0}(s) d s=A_{0}\langle T\rangle
$$

The second equality sign follows from integration by parts using $\frac{d}{d s} S_{0}(s)=$ $-P_{0}(s)$. 
For noise model $\mathrm{B}, P_{0}(s)$ is a gaussian $P_{0}(s)=\mathcal{G}_{\hat{\sigma}}\left(s-T_{0}\right)$ where $\hat{\sigma}$ is the width of the distribution and $T_{0}$ is the noise-free interval in the presence of the input potential $h_{0}$. Thus, for this noise model, $A_{0}=1 /\langle T\rangle=1 / T_{0}$ as in the noise-free case. This is a nice property of noise model B. For noise models $\mathrm{A}$ and $\mathrm{C}$, the mean interval, and hence $A_{0}$, changes with the level of noise.

7.2 Stability of Asynchronous Firing. In this subsection, the stability of incoherent firing is analyzed. We assume that for $t>0$, the activity is subject to a small perturbation around the fixed point at $A_{0}$,

$$
A(t)=A_{0}+\hat{A}_{1} e^{i \omega t+\lambda t},
$$

with $\hat{A}_{1} \ll A_{0}$. The perturbation in the activity induces a perturbation in the postsynaptic potential,

$$
h(t)=h_{0}+h_{1} e^{i \omega t+\lambda t}
$$

with $h_{0}=J_{0} \hat{\epsilon}(0) A_{0}$ and $h_{1}=J_{0} \hat{\epsilon}(\omega-i \lambda) \hat{A}_{1}$, where

$$
\hat{\epsilon}(\omega-i \lambda)=|\hat{\epsilon}(\omega-i \lambda)| e^{-i \psi(\omega-i \lambda)}=\int_{0}^{\infty} \epsilon_{0}(s) e^{-i \omega s-\lambda s} d s
$$

is a Laplacian transform of $\epsilon_{0}$. The Fourier transform is defined by equation 7.5 with $\lambda=0$. The angle $\psi($.) is the phase shift between $h$ and $A$.

The change in the potential causes some of the neurons to fire earlier (when the change in $h$ is positive) and others to fire later (whenever the change is negative). The perturbation may therefore build up ( $\lambda>0$, the incoherent state is unstable) or decay back to zero $(\lambda<0$, the incoherent state is stable). At the transition between the region of stability and instability, the amplitude of the perturbation remains constant $(\lambda=0$, marginal stability of the incoherent state). These transition points, defined by $\lambda=0$, are determined now.

We use equations 7.3 and 7.4 on the right-hand side of the linearized population equation, 5.9. After cancellation of a common factor $\hat{A}_{1} \exp (i \omega t)$, the result can be written in the form

$$
1-\hat{P}(\omega)=i \omega J_{0} A_{0} \hat{\epsilon}(\omega) \hat{\mathcal{L}}(\omega)
$$

$\hat{P}(\omega)$ is the Fourier transform of the interval distribution $P_{h_{0}}(t \mid \hat{t})$, and $\hat{\mathcal{L}}(\omega)$ is the transform of the filter $\mathcal{L}$ in Table 1. Equation 7.6 defines the bifurcation points where the incoherent firing state loses its stability toward an oscillation with frequency $\omega$.

So far the result is completely general. We now specify the model. For $\mathrm{SRM}_{0}$ neurons with gaussian noise in the reset (noise model B), the interval 


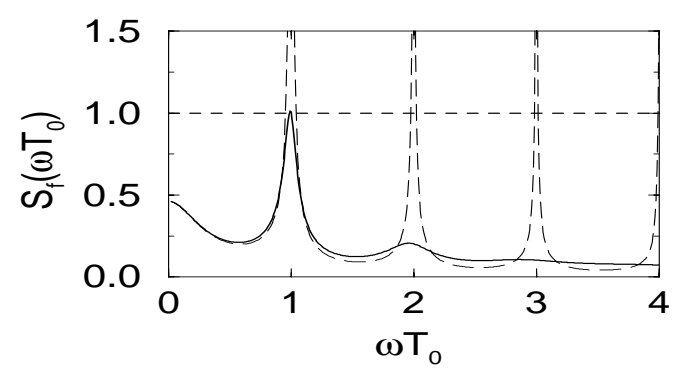

Figure 10: Amplitude condition for the bifurcation of an oscillatory solution. The feedback gain $S_{f}$ is plotted as a function of the normalized frequency $\omega T_{0}$ for two different values of the noise, $\sigma=1 \mathrm{~ms}$ (solid line) and $\sigma=0.1 \mathrm{~ms}$ (dashed line). Given a feedback with appropriate phase, instabilities of the incoherent firing state are possible for frequencies where $S_{f}>1$. For low noise, $S_{f}$ crosses unity (dotted horizontal line) at frequencies $\omega \approx \omega_{n}=n 2 \pi / T_{0}$. For $\sigma=1 \mathrm{~ms}$ there is a single instability region for $\omega T_{0} \approx 1$. The feedback gain is defined by the right-hand side of equation 7.7. For the plot we have set $T_{0}=2 \tau$.

distribution is a gaussian centered at $T_{0}$ and the filter is a $\delta$-function, $\mathcal{L}(x)=$ $\delta(x) / \eta^{\prime}$. Hence equation 7.6 is of the form

$$
1=\frac{i \omega}{\eta^{\prime}} \frac{J_{0} A_{0} \hat{\epsilon}(\omega)}{1-\hat{\mathcal{G}}_{\sigma}(\omega) e^{-i \omega T_{0}}},
$$

where $\hat{\mathcal{G}}_{\sigma}(\omega)=\exp \left\{-\frac{1}{2} \sigma^{2} \omega^{2}\right\}$ is the Fourier transform of a gaussian with width $\sigma$. We note that equation 7.7 could have been obtained directly from the signal gain, equation 6.1, after replacement of the input term $J^{\text {ext }} \hat{\tilde{\epsilon}}(\omega) \hat{\mathcal{I}}(\omega) \longrightarrow J_{0} \hat{\epsilon}(\omega) \hat{A}_{1}$ and evaluation of $\hat{P}(\omega)$ and $\hat{\mathcal{L}}(\omega)$ for noise model B. In the following we analyze solutions of equation 7.7. To do so we split the equation into two equations: one for the absolute value and the other for the phase. Let us start with the equation for the absolute values.

A necessary condition for a solution to equation 7.7 is a feedback gain $S_{f}$ of unity, where $S_{f}$ is defined by the absolute value of the right-hand side of the equation. In Figure 10 we have plotted $S_{f}$ as a function of $\omega T_{0}$. The left-hand side of equation 7.7 corresponds to a horizontal line at unity. Solutions to equation 7.7 may exist only for frequencies $\omega \approx \omega_{n}=n 2 \pi / T_{0}$ with integer $n$, where $T_{0}=1 / A_{0}$ is the typical interspike interval of the neurons. $A_{0}$ is determined by equation 7.2 , with $h_{0}$ given by equation 7.1. Thus $T_{0}=1 / A_{0}$ includes the effect of the feedback. $T_{0}$ is not the intrinsic frequency of the free neuron, which could be quite different.

A solution with $\omega \approx \omega_{1}$ implies that the period of the population activity is identical to the period of individual neurons driven by an input potential $h_{0}$. Higher harmonics correspond to cluster states: each neuron fires with 
a mean period of $T_{0}$, but neurons tend to fire in groups so that the activity oscillates several times faster. We see from Figure 10 that the harmonics are relevant for low noise only. At high-noise level, even the solution $\omega \approx \omega_{1}$ disappears. For $\sigma \rightarrow 0$ the absolute value of the denominator of equation 7.7 is $2\left|\sin \left(\omega T_{0} / 2\right)\right|$, and solutions may occur for all higher harmonics.

Figure 10 specifies the amplitude condition for the solution of equation 7.7. We now turn to the phase equation. In order to work out the phase conditions, we need the Fourier transform of the response kernel $\epsilon_{0}$ defined in equation 2.5. It is given by equation 7.5 with amplitude $|\hat{\epsilon}(\omega)|=$ $\left(1+\omega^{2} \tau^{2}\right)^{-1}$ and phase:

$$
\psi(\omega)=\omega \Delta^{\mathrm{ax}}+2 \arctan (\omega \tau) .
$$

We note that a change in the delay $\Delta^{\mathrm{ax}}$ affects only the phase of the Fourier transform, not the amplitude. By changing the transmission delay, we can therefore systematically shift the phase of the numerator on the right-hand side of equation 7.7, a convenient property that we will exploit below.

Since we know that solutions are possible only for $\omega \approx \omega_{n}$, we set $\omega=$ $\omega_{n}+v_{n}$. The numerical solutions of equation 7.7 for different values of the delay $\Delta^{\mathrm{ax}}$ and different levels of the noise $\sigma$ are shown in the bifurcation diagram in the center of Figure 11. Neurons interact with strength $J_{0}=1$. The value of the threshold $\vartheta$ was adjusted so that the mean interval in the presence of coupling was $T_{0}=2 \tau$. (For this choice of parameters, the interval of the free neuron would be about twice as long.) In the simulations shown in the four insets in Figure 11, we have taken $\tau=4 \mathrm{~ms}$ and a total number of $N=1000$ neurons.

7.3 Bifurcation Diagram. Let us consider a network with interaction delay $\Delta^{\mathrm{ax}}=2 \mathrm{~ms}$. This corresponds to an $x$-value of $\Delta^{\mathrm{ax}} / T_{0}=0.25 \mathrm{in}$ Figure 11. The phase diagram predicts that at a noise level of $\sigma=0.5 \mathrm{~ms}$, the network is in a state of asynchronous firing. The activity during a simulation run is shown in the inset in the upper right-hand corner. It confirms that the activity fluctuates around a constant value of $A_{0}=1 / T_{0}=0.125 \mathrm{kHz}$.

If the noise level of the network is significantly reduced, the system crosses the short-dashed line. The line is the boundary at which the constant activity state becomes unstable with respect to an oscillation with $\omega \approx 3\left(2 \pi / T_{0}\right)$. A simulation result for a network at a noise level of $\sigma=0.1$ but otherwise the same parameters as before confirms that the population activity exhibits an oscillation with period $T^{o s c} \approx T_{0} / 3 \approx 2.6 \mathrm{~ms}$.

We now return to our original parameters of $\sigma=0.5 \mathrm{~ms}$ and $\Delta^{\text {ax }}=2 \mathrm{~ms}$ and reduce the axonal transmission delay while keeping the noise level fixed. This correponds to a horizontal move across the phase diagram in Figure 11. At some point, the system crosses the solid line, which marks the transition to an instability with frequency $\omega_{1}=2 \pi / T_{0}$. Again this is confirmed by the simulation results shown in the inset in the upper left 


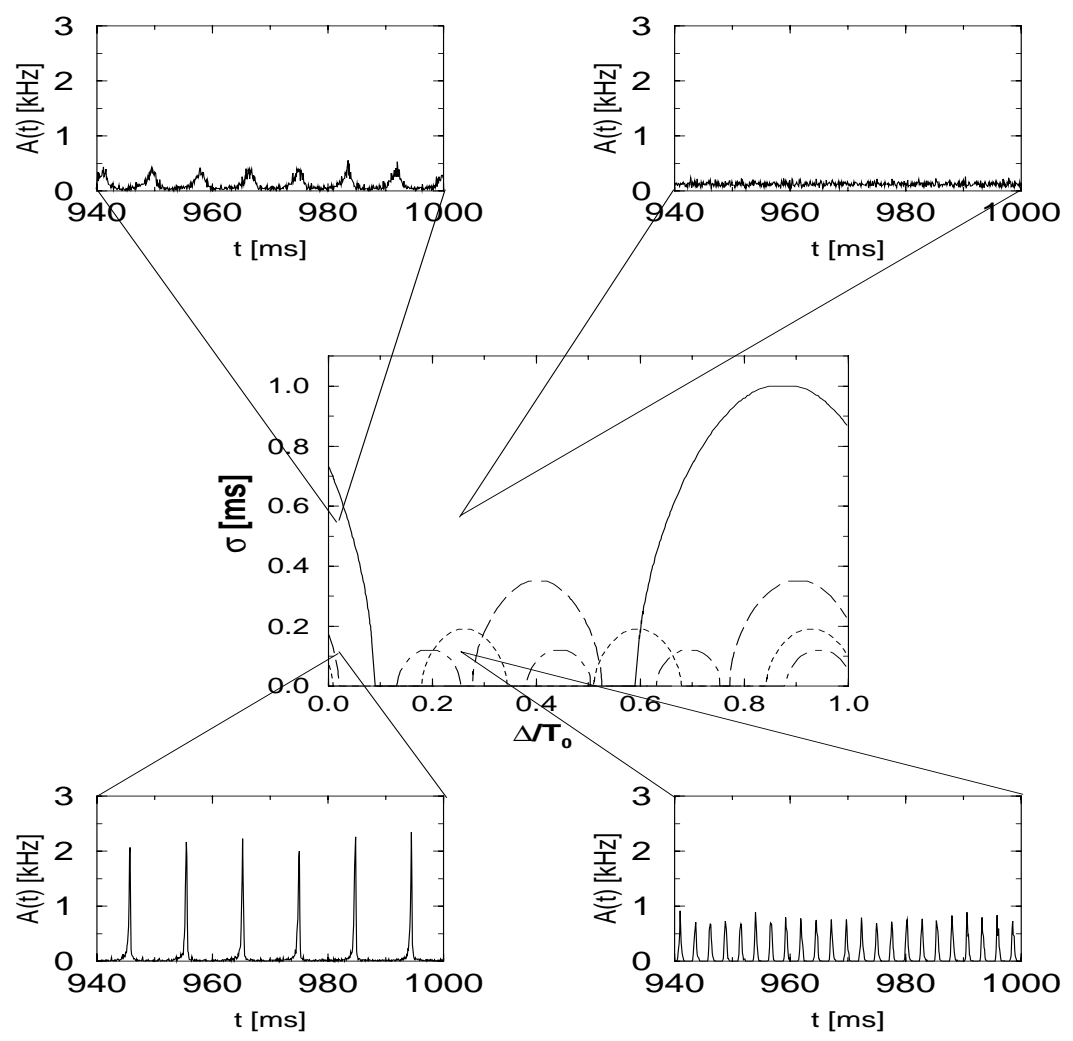

Figure 11: Stability diagram (center) as a function of noise $\sigma$ (y-axis) and delay $\Delta^{\mathrm{ax}}(x$-axis). The diagram shows the borders of the stability region with respect to $\omega_{1}, \ldots, \omega_{4}$. For high values of the noise, the asynchronous firing state is always stable. If the noise is reduced, the asynchronous state becomes unstable with respect to an oscillation with frequency $\omega_{1}$ (solid border lines,) or $\omega_{2}$ (longdashed border lines), or $\omega_{3}$ (short-dashed border lines), or $\omega_{4}$ (long-short dashed border lines). Four insets show typical patterns of the activity as a function of time taken from a simulation with 1000 neurons. Parameters $\sigma=0.5 \mathrm{~ms}$ and $\Delta^{\mathrm{ax}}=0.2 \mathrm{~ms}$ (top left); $\sigma=0.5 \mathrm{~ms}$ and $\Delta^{\mathrm{ax}}=2.0 \mathrm{~ms}$ (top right); $\sigma=0.1 \mathrm{~ms}$ and $\Delta^{\mathrm{ax}}=0.2 \mathrm{~ms}$ (bottom left); $\sigma=0.1 \mathrm{~ms}$ and $\Delta^{\mathrm{ax}}=2.0 \mathrm{~ms}$ (bottom right); Since the pattern repeats along the $x$-axis with period $T_{0}$, we have plotted a normalized delay $\Delta^{\mathrm{ax}} / T_{0}$.

corner. If we now decrease the noise level, the oscillation becomes more pronounced (see the bottom right of Figure 11).

In the limit of low noise, the incoherent network state is unstable for virtually all values of the delay. The region of the phase diagram in Figure 11 
around $\Delta^{\mathrm{ax}} / T_{0} \approx 0.1$ looks stable but hides instabilities with respect to the higher harmonics $\omega_{6}$ and $\omega_{5}$, which are not shown. We emphasize that the specific location of the stability borders depends on the form of the postsynaptic response function $\epsilon$. The qualitative features of the phase diagram in Figure 11 are generic and hold for all kinds of response kernels.

The numerical results apply to the response kernel $\epsilon_{0}(s)$ defined in equation 2.5, which corresponds to a synaptic current $\alpha(s)$ with zero rise time (cf. equations 2.9 and 2.8). What happens if $\alpha$ is a double exponential with rise time $\tau_{\text {rise }}$ and decay time $\tau_{\text {syn }}$ ? In this case, the right-hand side of equation 7.7 has an additional factor $\left[1+i \omega \tau_{\text {rise }}\right]^{-1}$ that leads to two changes. First, due to the reduced amplitude of the feedback, instabilities with frequencies $\omega>\tau_{\text {rise }}^{-1}$ are suppressed. The tongues for the higher harmonics are therefore smaller. Second, the phase of the feedback changes. Thus, all tongues of frequency $\omega_{n}$ are moved horizontally along the $x$-axis by an amount $\Delta / T_{0}=-\operatorname{atan}\left(\omega_{n} \tau_{\text {rise }}\right) /(n 2 \pi)$.

What happens if the excitatory interaction is replaced by inhibitory coupling? A change in the sign of the interaction correponds to a phase shift of $\pi$. For each harmonic, the region along the delay axis where the incoherent state is unstable for excitatory coupling (cf. Figure 11) becomes stable for inhibition, and vice versa. In other words, we simply have to shift the instability tongues for each frequency $\omega_{n}$ horizontally by an amount $\Delta / T_{0}=1 /(2 n)$. Otherwise the pattern remains the same.

\section{Discussion}

8.1 Assumptions. We have discussed an integral equation for the population dynamics. The validity of the population equations relies on three assumptions: (1) a homogeneous population of (2) an infinite number of neurons that show (3) no adaptation.

It is clear that there are no large and completely homogeneous populations in biology. The population equations may nevertheless be a useful starting point for a theory of heterogeneous populations (Tsodyks, Mitkov, \& Sompolinsky, 1993; Senn et al., 1996; Chow, 1998; Pham et al., 1998; Brunel \& Hakim, 1998). We expect that most of the results carry over to nonhomogeneous networks. In a sense, noise model B can be considered an annealed version of a heterogeneous model where the reset value varies from one neuron to the next. The treatment of heterogeneity as noise (reset values are randomly chosen after each reset rather than only once at the beginning) neglects, however, correlations that would be present in a truly heterogeneous model. To replace a heterogenous model with a noisy version of a homogeneous model is somewhat ad hoc but common practice in the literature. Wilson and Cowan (1972), for example, motivated the sigmoidal threshold function in their model by a heterogeneous system where the threshold value varies from one neuron to the next. In or- 
der to analyze the system, they neglect correlations and replace the heterogeneous system by a homogeneous population with noisy threshold (noise model A). A more recent example for the replacement of a heterogeneous system by a noisy homogeneous system can be found in Pham et al., 1988.

A generalization of equations 3.3 and 3.7 to a network subdivided into several pools is possible. Within each pool, neurons are homogeneous. The activity of a pool $m$ is $A_{m}(t)$. A neuron in pool $m$ receives input from all neurons in pool $n$ with strength $w_{m n}=J_{m n} / N_{n}$. The postsynpatic potential of neurons in pool $m$ is then given by a straightforward generalization of equation 3.3 (Gerstner 1995) $h_{m}(t \mid \hat{t})=\sum_{n} J_{m n} \int_{0}^{\infty} \epsilon(t-\hat{t}, s) A_{n}(t-s) d s$. The population equation 3.7 remains unchanged but has to be applied to each pool activity $A_{m}$ separately. The pools are coupled via the potential $h_{m}$, which determines the kernel $P_{h_{m}}(t \mid \hat{t})$. A transition from discrete pools to a continuous population is possible (Gerstner, 1995) and might be useful for modeling of primary visual cortex.

The second condition is the limit of a large network. For $N \rightarrow \infty$ the population activity shows no fluctuations, and this fact has been used for the derivation of the population equation. For systems of finite size, fluctuations are important since they limit the amount of information that can be transmitted by the population activity. For populations without internal coupling $\left(J_{0}=0\right)$, fluctuations can be calculated directly from the interval distribution $P_{h}(t \mid \hat{t})$. For networks with internal coupling, an exact treatment of finite size effects is difficult. For noise model A, first attempts toward a description of the fluctuations have been made (Spiridon, Chow, \& Gerstner, 1998). For noise model C, finite size effects in the low-connectivity limit are treated in Brunel and Hakim (1999).

The limit of no adaptation seems to be valid for fast spiking neurons (Connors, 1990). Most cortical neurons, however, show adaptation. A generalization of the population equations to neuron models with adaptation does not seem straightforward. From the modeling point of view, all IF neurons are in the class of nonadaptive neurons, since the membrane potential is reset (and the past forgotten) after each output spike. Both leaky and nonleaky IF neurons can therefore be mapped to a response kernel description with no dependence on earlier output spikes (Gerstner, 1995). To a very good approximation, the Hodgkin-Huxley model can also be mapped to a response kernel description (Kistler et al., 1997). The condition of short memory (that is, no adaptation) leads to the class of renewal models (Perkel, Gerstein, \& Moore, 1967; Stein, 1967; Cox, 1962), and this is where the integral equation applies; (cf. Gerstner, 1995).

8.2 Signal Transmission by Incoherent Firing. We have mainly focused on the state of incoherent firing in the low-noise regime. This state may be particularly interesting for information transmission, since the system 
can respond rapidly to changes in the input current. For noise model B, the signal gain defined as the amplitude of the population activity divided by that of the input current shows no cutoff at high frequencies (Knight, 1972a). The effective cutoff frequency of the system is therefore given by the input current. Changes in the input current are, of course, limited by the opening and closing times of synaptic channels. Each presynpatic spike evokes a postsynaptic current pulse of finite width. The time course of the current pulse determines the response time of the system (Treves, 1993).

These insights may have important implications for modeling as well as for interpretations of experiments. It is often thought, that the response time of neurons is directly related to the membrane time constant $\tau_{m}$. This idea has been criticized repeatedly since in some cases neurons do respond much faster (Treves, 1992; Koch, Rapp, \& Segev, 1996). In neural network modeling, a description of the population activity by a differential equation of the form 1.2 is common practice. Our results suggest that the time constant $\tau$ of the left-hand side of equation 1.2 should be of the order of the duration of the synaptic current pulse.

We have seen that the Wilson-Cowan integral equation 1.3 and hence the differential equation 1.2 describes neurons with absolute refractoriness and noise model $\mathrm{A}$; the gain function $g$ in equation 1.3 may be identified with the escape rate $f$. For neurons with absolute refractoriness and noise model A, the filter $\mathcal{L}$ is a low-pass filter with cutoff frequency $f\left(h_{0}\right)$ (cf. appendix E). If the mean activity of the system is low, then the cutoff freqency is low, and the activity follows during a transient the input potential $h$. The transient is therefore slow. On the other hand, if the rate $f\left(h_{0}\right)$ is high, then the activity follows the derivative $h^{\prime}$, and the transient is fast. Thus time-coarse graining would be possible with a time constant smaller than $f\left(h_{0}\right)^{-1}$. We must keep in mind that even if we take a short integration time constant, the differential equation 1.2 is always a description of noise model A. We hope that the treatment presented in this article helps to clarify some important points. The limitations of the differential equation 1.2 are already mentioned in Wilson and Cowan (1972) and have been discussed in Abbott and van Vreeswijk (1993), Treves (1993), and Gerstner (1995). For noise model A, the integral equation can be replaced by a system of differential equations (Eggert \& van Hemmen, 1997) or by partial differential equations (Gerstner \& van Hemmen, 1992). For noise model $\mathrm{C}$, partial differential equations for the distribution of the membrane potential $u$ have been developed (Abbott \& van Vreeswijk, 1993; Brunel \& Hakim, 1998) and are an alternative to the approach by integral equations.

Since we consider incoherent firing as a state with useful signal transmission properties, we must be concerned about the stability of such a state. Incoherent firing can be stabilized by a suitable choice of time constants, transmission delay, and noise (Abbott \& van Vreeswijk, 1993; Gerstner \& 
van Hemmen, 1993, 1994; Gerstner, 1995). For low noise, incoherent states are unstable for nearly all choices of parameters. Instabilities lead toward oscillations, either with a period comparable to the typical interval of the neuronal spike trains, or much faster (higher harmonics) (Golomb, Hansel, Shraiman, \& Sompolinsky, 1992; Gerstner \& van Hemmen, 1993; Ernst, Pawelzik, \& Geisel, 1994; Golomb \& Rinzel, 1994). The harmonics have also been called cluster states since neurons spontaneously split into groups of neurons that fire approximately together. Higher harmonics can be easily suppressed by noise.

The instability of asynchronous firing, has previously been been studied in phase models (Kuramoto, 1975, 1984; Winfree, 1980; Ermentrout, 1981; Strogatz \& Mirollo, 1991; Golomb et al., 1992). Phase models can describe arbitrary nonlinear oscillator systems in the limit of weak coupling (Ermentrout, 1981). In contrast to phase models, the analytical framework presented in this article does not rely on a weak coupling assumption but works just as well for strong coupling. The mean interspike interval $T_{0}$ in the asynchronous or synchronous state is determined self-consistently and includes the effects of the feedback from other neurons. In the absence of coupling, the model neurons may be silent or exhibit a Poisson-like rather than an oscillatory firing behavior. Thus neurons are not necessarily oscillators. To analyze the stability in the Poisson regime, we simply have to choose the appropriate interval distribution $P_{h_{0}}(t \mid \hat{t})$, determine its Fourier transform $\hat{P}(\omega)$, and the filter $\hat{\mathcal{L}}(\omega)$, and put the expressions in the bifurcation equation, 7.6.

The instability of asynchronous firing in pulse-coupled neuron models has been studied in the noise-free case (Abbott \& van Vreeswijk, 1993; Gerstner \& van Hemmen, 1993; Gerstner, 1995). The effect of small inhomogeneities has been studied by Tsodyks et al. (1993) and a network with several populations and types on ion currents by Treves (1993). Noise in the differential equation of delayless IF neurons was treated by Kuramoto (1991) and Abbott and van Vreeswijk (1993). An analysis similar to that of Abbott and van Vreeswijk (1993), but for heterogeneous networks with low connectivity, has been done by Brunel and Hakim (1999). The effect of threshold noise (noise model A) in the spike response model including delays has been investigated by Gerstner (1995). For the analysis in section 7, we have assumed noise in the reset (noise model B). This noise model is probably not the most realistic one, but it is particularly convenient to work with since the mean interspike interval is independent of the noise level. For other noise models, a bifurcation diagram as in Figure 11 will look more complicated since the mean interval depends on the noise level. A way out would be to readjust the threshold for each noise level so as to keep the mean interval constant.

If the network is firing incoherently, then the population activity responds immediately to an abrupt change in the input (Treves, 1992; Tsodyks \& Se- 
jnowski, 1995; van Vreeswijk \& Sompolinsky, 1996; Horn \& Levanda, 1998). There is no integration delay. In the case of incoherent firing, there are always some neurons close to threshold, and this is why the system as a whole can respond immediately. This property suggests that a population of neurons may transmit information fast and reliably. Fast information processing seems to be a necessary requirement for biological nervous systems if the reaction time experiments are to be accounted for (Thorpe et al., 1996).

\section{Appendix A: Wilson-Cowan Integral Equations}

We apply the population equation 3.7 to $\mathrm{SRM}_{0}$ neurons with noise model A. The escape rate is a sigmoidal function $f[u]$ and $\vartheta=0$. The neuron model is specified by a refractory function $\eta$ in three pieces. First, during an absolute refractory time $0<s \leq \delta^{\text {abs }}$, we formally set $\eta(s)$ to $-\infty$. Second, during the relative refractory period $\delta^{\text {abs }}<s<\delta^{\text {rel }}, \eta$ may take some arbitrary values $\eta(s)$. Third, $\eta(s)=0$ for $s \geq \delta^{\text {rel }}$.

Given $\eta$ it seems natural to split the integral in the activity equation 3.7 into three pieces:

$$
\begin{aligned}
A(t)= & \int_{-\infty}^{t-\delta^{\text {rel }}} P_{h}(t \mid \hat{t}) A(\hat{t}) d \hat{t}+\int_{t-\delta^{\text {rel }}}^{t-\delta^{\text {abs }}} P_{h}(t \mid \hat{t}) A(\hat{t}) d \hat{t} \\
& +\int_{t-\delta^{\mathrm{abs}}}^{t} P_{h}(t \mid \hat{t}) A(\hat{t}) d \hat{t} .
\end{aligned}
$$

The interval distribution $P_{h}(t \mid \hat{t})$ for noise model A is given by equation 2.15, and is repeated here for convenience:

$$
P_{h}(t \mid \hat{t})=f[h(t)+\eta(t-\hat{t})] \exp \left\{-\int_{\hat{t}}^{t} f\left[h\left(t^{\prime}\right)+\eta\left(t^{\prime}-\hat{t}\right)\right] d t^{\prime}\right\} .
$$

The last term in equation A.1 vanishes since spiking is impossible during the absolute refractory time, $f[-\infty]=0$. In the first term we can move a factor $f[h(t)+\eta(t-\hat{t})]=f[h(t)]$ in front of the integral since $\eta$ vanishes for $t-\hat{t}>\delta^{\text {rel }}$. The first term in equation A.1 can therefore be rewritten as

$$
f[h(t)] \int_{-\infty}^{t-\delta^{\text {rel }}} S_{h}(t \mid \hat{t}) A(\hat{t}) d \hat{t}
$$

where we have used the definition (see equation 2.14) of the survivor function in noise model A. The integral in equation A. 3 can be treated further if we use the normalization given in equation 3.6. The result of these manipulations is

$$
A(t)=f[h(t)]\left[1-\int_{t-\delta^{\text {rel }}}^{t} S_{h}(\hat{t t}) A(\hat{t}) d \hat{t}\right]+\int_{t-\delta^{\text {rel }}}^{t-\delta^{\text {abs }}} P_{h}(\hat{t t}) A(\hat{t}) d \hat{t}
$$


Except for a slight change of notation, ${ }^{2}$ equation A.4 is identical to the integral equation derived by Wilson and Cowan (1972). It applies to neurons with relative refractoriness of finite duration. For $\delta^{\text {rel }} \rightarrow \infty$ the expression in the square brackets vanishes, and we are back to equation 3.7.

If there is no relative refractoriness but absolute refractoriness only, we may set $\delta^{\text {rel }}=\delta^{\text {abs }}$. The last term in equation A.4 then vanishes. Furthermore, during the absolute refractory period, we have a survival probablity $S_{h}(t \mid \hat{t})=1$ since the neurons cannot fire. This yields

$$
A(t)=f[h(t)]\left\{1-\int_{t-\delta_{\text {abs }}}^{t} A\left(t^{\prime}\right) d t^{\prime}\right\},
$$

which is after the substitution $f \longrightarrow g$ exactly the Wilson-Cowan integral equation 1.3.

\section{Appendix B: Compression of Firing Times}

We prove the following generalization of the locking theorem:

1. $\partial_{t} h+\partial_{\hat{t}} h>0$ is a necessary condition for a compression of firing times.

2. For IF neurons, the condition $\partial_{t} h+\partial_{\hat{t}} h>0$ is also sufficient.

Proof. With view to equations 4.2 and 4.3 , we need to show that $\partial_{t} h+\partial_{\hat{t}} h<$ $0 \Longrightarrow C<0$. We will use that $u^{\prime}=\eta^{\prime}+\partial_{t} h>0$ at the moment of firing. The assertion follows from

$$
C=\frac{\partial_{t} h+\partial_{\hat{t}} h}{\eta^{\prime}-\partial_{\hat{t}} h}=\frac{\partial_{t} h+\partial_{\hat{t}} h}{u^{\prime}-\left[\partial_{t} h+\partial_{\hat{t}} h\right]} .
$$

We need to show that for IF neurons the denominator in equation B.1 is always positive. With view to equation 4.5 , this is equivalent to the statement $\tilde{\eta}_{0}+h\left(t-T_{b}\right)+\tau h^{\prime}\left(t-T_{b}\right)>0$ where $t-T_{b}=\hat{t}$ is the last firing time. Hence $u^{\prime}(\hat{t})>0$ and $u(\hat{t})=\vartheta$. The differential equation for the IF model, 2.8, is $\tau u^{\prime}=-u+$ input where input comprises synaptic and external currents. The input potential $h(t)$ is a solution to the differential equation-hence, input $=h+\tau h^{\prime}$

$$
0<\tau u^{\prime}(\hat{t})=-\vartheta+\text { input }<-u_{\text {reset }}+h+\tau h^{\prime},
$$

where we have used $u_{\text {reset }}<\vartheta$. The assertion follows since $\tilde{\eta}_{0}=-u_{\text {reset }}$.

\footnotetext{
${ }^{2}$ In Wilson and Cowan (1972), $f$ is called $\mathcal{S}, S_{h}(t \mid \hat{t}) A(\hat{t})$ is called $R(t, \hat{t})$, and $P_{h}(t \mid \hat{t}) A(\hat{t})$ is $\mathcal{S} R(t, \hat{t})$.
} 
Appendix C: Locking in the Presence of Noise for $\mathrm{SRM}_{0}$ Neurons

We start from equation 4.12 and search for periodic pulse-type solutions. We assume that the pulses are gaussians with width $d$ and repeat with period $T$ : $A(t)=\sum_{n} \mathcal{G}_{d}(t-n T)$. The pulse width $d$ will be determined self-consistently from equation 4.12. The integral over $r$ in that equation can be performed and yields a gaussian with width $\tilde{\sigma}=\left[d^{2}+\sigma^{2}\right]^{1 / 2}$. Equation 4.12 becomes

$$
\sum_{n} \mathcal{G}_{d}(t-n T)=\left[1+\frac{h^{\prime}(t)}{\eta^{\prime}(T)}\right] \sum_{n} \mathcal{G}_{\tilde{\sigma}}\left[t-T_{b}(t)-n T\right],
$$

where $T_{b}(t)=\tau \ln \left\{\tilde{\eta}_{0} /[h(t)-\vartheta]\right\}$ is the interspike interval looking backward in time.

Let us work out the self-consistency condition and focus on the pulse around $t \approx 0$. It corresponds to the $n=0$ term on the left-hand side, which must equal the $n=-1$ term on the right-hand side of equation C.1. We assume that the pulse width is small $d \ll T$ and expand $T_{b}(t)$ to linear order around $T_{b}(0)=T$. This yields

$$
t-T_{b}(t)=t\left[1+\frac{h^{\prime}(0)}{\eta^{\prime}(T)}\right]-T .
$$

The expansion is valid if $h^{\prime}(t)$ varies slowly over the width $d$ of the pulse. We use equation C.2 in the argument of the gaussian on the right-hand side of equation C.1. Since we have assumed that $h^{\prime}$ varies slowly, the factor $h^{\prime}(t)$ in equation C.1 may be replaced by $h^{\prime}(0)$. In the following we suppress the arguments and write simply $h^{\prime}$ and $\eta^{\prime}$. The result is

$$
\mathcal{G}_{d}(t)=\left(1+\frac{h^{\prime}}{\eta^{\prime}}\right) \mathcal{G}_{\tilde{\sigma}}\left[t\left(1+\frac{h^{\prime}}{\eta^{\prime}}\right)\right] .
$$

The gaussian on the left-hand side of equation C.3 must have the same width as the gaussian on the right-hand side. The condition is $d=\tilde{\sigma} /\left[1+h^{\prime} / \eta^{\prime}\right]$ with $\tilde{\sigma}=\left[d^{2}+\sigma^{2}\right]^{1 / 2}$. A simple algebraic transformation yields an explicit expression for the pulse width,

$$
d=\sigma\left[2\left(h^{\prime} / \eta^{\prime}\right)+\left(h^{\prime} / \eta^{\prime}\right)^{2}\right]^{-1 / 2},
$$

where $d$ is the width of the pulse and $\sigma$ is the strength of the noise.

Appendix D: The Filter $\mathcal{L}(x)$ for Noise Model B

We calculate the linear response of IF neurons with noise model $B$ to an input $\Delta \mathcal{I}$. We start from equation 4.11 and formally integrate over the variable $\hat{t}$. 
This yields

$$
A(t)=\int d r\left[1+\frac{d}{d \hat{t}} T(\hat{t}, r)\right]^{-1} \mathcal{G}_{\sigma}(r) A\left[t-T_{b}(t, r)\right],
$$

where $T_{b}(t, r)$ is the backward interval of neurons that have been reset the last time with value $r$. The derivative has to be evaluated at $\hat{t}=t-T_{b}(t, r)$. In order to calculate the derivative needed in equation D.1, we write the membrane potential in the form

$$
u(t)=-\tilde{\eta}_{0} e^{r / \tau} \exp \left(-\frac{t-\hat{t}}{\tau}\right)+h(t)-h(\hat{t}) \exp \left(-\frac{t-\hat{t}}{\tau}\right),
$$

where we have used equations 2.1, 2.4, 2.10, and 2.16. Neurons that fire at $t$ have $u(t)=\vartheta$, hence

$$
\vartheta=-\tilde{\eta}_{0} e^{r / \tau} \exp \left(-\frac{T(\hat{t}, r)}{\tau}\right)+h[\hat{t}+T(\hat{t}, r)]-h(\hat{t}) \exp \left(-\frac{T(\hat{t}, r)}{\tau}\right) .
$$

We take the derivative of equation D. 3 with respect to $\hat{t}$ and find

$$
\frac{d}{d \hat{t}} T(\hat{t}, r)=-\frac{1}{u^{\prime}}\left\{h^{\prime}(t)-h^{\prime}\left[t-T_{b}(t, r)\right] e^{-T_{b}(t, r) / \tau}\right\},
$$

where $u^{\prime}$ is the derivative of equation D.2.

We now linearize equation D.1. The variation $\Delta \mathcal{I}$ in the input current causes a perturbation $\Delta h(t)$ of the input potential (see equation 2.4) around $h_{0}$, which in turn evokes a change $\Delta A(t)$ in the activity. We set $h(t)=h_{0}+$ $\Delta h(t)$ and $A(t)=A_{0}+\Delta A(t)$ (where $\Delta A$ is of the order $\Delta h$ ) and expand both sides of equation D.1 to first order in $\Delta A$ or $\Delta h$. Note that the zero-order term of $\frac{d}{d \hat{t}} T(\hat{t}, r)$ vanishes (see equation D.4). The result of the linearization is

$$
\Delta A(t)=\int \mathcal{G}_{\sigma}(r) \Delta A[t-T(r)] d r-A_{0} \int \mathcal{G}_{\sigma}(r) \frac{d}{d \hat{t}} T(\hat{t}, r) d r,
$$

where $T(r)=T_{0}+T_{1} r$ is the interval of a neuron in the asynchronous state (no perturbation), which has been reset with value $r . T_{1}=\tilde{\eta}_{0} /\left(\tilde{\eta}_{0}+h_{0}\right)$ has been introduced after equation 2.18. $\frac{d}{d \hat{t}} T(\hat{t}, r)$ is given by equation D.4 with $u^{\prime}=\tau^{-1}\left[\tilde{\eta}_{0} \exp (r / \tau)+h_{0}\right] \exp [-T(r) / \tau]$. To simplify the expression, $u^{\prime}$ may be approximated by its value for $r=0$ and moved in front of the integral. Similarly, we use $\exp \left[-T_{b}(\hat{t}, r] / \tau\right] \approx \exp \left[-T_{0} / \tau\right)$. The final result is

$$
\begin{aligned}
\Delta A(t)= & \int \mathcal{G}_{\hat{\sigma}}(r) \Delta A\left(t-T_{0}-r\right) d r \\
& +\frac{A_{0}}{u^{\prime}}\left[h^{\prime}(t)-e^{-T_{0} / \tau} \int \mathcal{G}_{\hat{\sigma}}(r) h^{\prime}\left(t-T_{0}-r\right) d r\right],
\end{aligned}
$$


where $\hat{\sigma}=T_{1} \sigma$. The first term contains the convolution of $\Delta A$ with the (unperturbed) interval distribution. The second term can be written as a filter $\mathcal{L}_{B}^{\mathrm{IF}}$ applied to $h^{\prime}$ (see Table 1 and equation 5.9).

\section{Appendix E: The Filter $\mathcal{L}(x)$ for Noise Model A}

In noise model A, we have from equation 2.14,

$$
S_{h}(t \mid \hat{t})=\exp \left\{-\int_{\hat{t}}^{t} f\left[\eta\left(t^{\prime}-\hat{t}\right)+h_{\mathrm{PSP}}\left(t^{\prime} \mid \hat{t}\right)\right] d t^{\prime}\right\}
$$

where $f[u]$ is the instantaneous escape rate across the noisy threshold. We write $h_{\mathrm{PSP}}(t \mid \hat{t})=h_{0}(t-\hat{t})+h_{1}(t \mid \hat{t})$. Taking the derivative with respect to $h_{1}$ yields

$$
\left.\frac{\partial S_{h}(\hat{t} \hat{t})}{\partial h_{1}\left(t_{1} \hat{t}\right)}\right|_{h_{1}=0}=-\mathcal{H}\left(t_{1}-\hat{t}\right) \mathcal{H}\left(t-t_{1}\right) f^{\prime}\left[\eta\left(t_{1}-\hat{t}\right)+h_{0}\left(t_{1}-\hat{t}\right)\right] S_{0}(t-\hat{t}),
$$

where $S_{0}(t-\hat{t})=S_{h_{0}}(t \mid \hat{t})$ and $f^{\prime}=d f / d u$. For $\mathrm{SRM}_{0}$ neurons, we have $h_{0}(t-\hat{t}) \equiv h_{0}$ and $h_{1}(t \mid \hat{t})=h_{1}(t)$, independent of $\hat{t}$. The filter $\mathcal{L}$ is therefore

$$
\mathcal{L}_{A}^{\mathrm{SRM}}\left(t-t_{1}\right)=\mathcal{H}\left(t-t_{1}\right) \int_{-\infty}^{t_{1}} d \hat{t} f^{\prime}\left[\eta\left(t_{1}-\hat{t}\right)+h_{0}\right] S_{0}(t-\hat{t}),
$$

as noted in Table 1.

It is now shown that equation E.3 can be reduced to

$$
\mathcal{L}_{A}^{\mathrm{SRM}}(x)=a \rho e^{-\rho x} \mathcal{H}(x),
$$

in two limiting cases: (1) $\mathrm{SRM}_{0}$-neurons and noise model A with instantaneous rate $f(u)=\rho \mathcal{H}(u-\vartheta)$ and arbitrary $\eta$ and $\epsilon$ kernels and (2) $\mathrm{SRM}_{0}$ neurons and noise model A with arbitrary $f(u)$ for neurons with absolute refractoriness $\eta(s)=-\infty$ for $0<s<\delta^{\text {abs }}$ and zero otherwise. Furthermore it is shown that for IF neurons with step function threshold,

$$
\mathcal{L}_{A}^{\mathrm{IF}}(x)=\mathcal{L}_{A}^{\mathrm{SRM}}(x)-e^{-T_{0} / \tau} \mathcal{L}_{A}^{\mathrm{SRM}}\left(x-T_{0}\right) .
$$

E.1 Step Function Threshold. We take $f(u)=\rho \mathcal{H}(u-\vartheta)$. For $\rho \rightarrow \infty$ neurons fire immediately as soon as $u(t)>\vartheta$, and we are back to the noisefree sharp threshold. For finite $\rho$, neurons respond stochastically with time constant $\rho^{-1}$.

Let us denote by $T_{0}$ the time between the last firing time $\hat{t}$ and the formal threshold crossing, $T_{0}=\min \left\{t-\hat{t} \mid \eta(t-\hat{t})+h_{0}=\vartheta\right\}$. The derivative of $f$ is a $\delta$-function

$$
f^{\prime}\left[\eta(t-\hat{t})+h_{0}\right]=\rho \delta\left[\eta(t-\hat{t})+h_{0}-\vartheta\right]=\frac{\rho}{\eta^{\prime}} \delta\left(t-\hat{t}-T_{0}\right),
$$


where $\eta^{\prime}=\left.\frac{d}{d s} \eta\right|_{T_{0}}$. The survivor function $S_{0}(s)$ is unity for $s<T_{0}$ and $S_{0}(s)=\exp \left[-\rho\left(s-T_{0}\right)\right]$ for $s>T_{0}$. Integration of equation E.3 yields

$$
\mathcal{L}_{A}\left(t-t_{1}\right)=\frac{1}{\eta^{\prime}} \mathcal{H}\left(t-t_{1}\right) \rho \exp \left[-\rho\left(t-t_{1}\right)\right]
$$

as claimed above. For the filter $\mathcal{L}_{A}^{\mathrm{IF}}$ we also have to evaluate the integral

$$
S_{0}(x) \int_{0}^{x} d \xi e^{-\xi / \tau} f^{\prime}\left[u\left(\xi^{\prime}\right)\right]=S_{0}(x) \mathcal{H}\left(x-T_{0}\right) \frac{\rho}{\eta^{\prime}} e^{-T_{0} / \tau}
$$

which confirms the assertion.

E.2 Absolute Refractoriness. We take an arbitrary escape rate $f(u) \geq 0$ with $\lim _{u \rightarrow-\infty} f(u)=0=\lim _{u \rightarrow-\infty} f^{\prime}(u)$. Absolute refractoriness is defined by a refractory kernel $\eta(s)=-\infty$ for $0<s<\delta^{\text {abs }}$ and zero otherwise. This yields $f\left[\eta(t-\hat{t})+h_{0}\right]=f\left(h_{0}\right) \mathcal{H}\left(t-\hat{t}-\delta^{\text {abs }}\right)$ and hence

$$
f^{\prime}\left[\eta(t-\hat{t})+h_{0}\right]=f^{\prime}\left(h_{0}\right) \mathcal{H}\left(t-\hat{t}-\delta^{\mathrm{abs}}\right) .
$$

The survivor function $S_{0}(s)$ is unity for $s<\delta^{\text {abs }}$ and decays as $\exp \left[-f\left(h_{0}\right)(s-\right.$ $\left.\delta^{\text {abs }}\right)$ ] for $s>\delta^{\text {abs }}$. Integration of equation E.3 yields

$$
\mathcal{L}_{A}\left(t-t_{1}\right)=\mathcal{H}\left(t-t_{1}\right) \frac{f^{\prime}\left(h_{0}\right)}{f\left(h_{0}\right)} \exp \left[-f\left(h_{0}\right)\left(t-t_{1}\right)\right]
$$

Note that for neurons with absolute refractoriness, the transition to the noiseless case is not meaningful. As shown in appendix $\mathrm{A}$, absolute refractoriness leads to the Wilson-Cowan integral equation, 1.3. Thus $\mathcal{L}_{A}=\mathcal{L}^{\text {WC }}$ defined in equation E.10 is the filter relating to equation 1.3. It is a low-pass filter with cutoff frequency $f\left(h_{0}\right)$, which depends on the input potential $h_{0}$.

\section{Acknowledgments}

I thank Carson C. Chow for many discussions, in particular for the idea of formulating the population equation as a conservation law. Thanks are due to the referees for numerous constructive comments. Carl van Vreeswijk has helped to improve the article through his many insightful suggestions.

\section{References}

Abbott, L. F., \& van Vreeswijk, C. (1993). Asynchronous states in a network of pulse-coupled oscillators. Phys. Rev. E, 48, 1483-1490.

Amari, S. (1974). A method of statistical neurodynamics. Kybernetik, 14, 201-215. 
Bauer, H. U., \& Pawelzik, K. (1993). Alternating oscillatory and stochastic dynamics in a model for a neuronal assembly. Physica $D, 69,380-393$.

Brunel, N., \& Hakim, V. (1999). Fast global oscillations in networks of integrateand-fire neurons with low firing rates. Preprint submitted to Neural Computation.

Chow, C. C. (1998). Phase-locking in weakly heterogeneous neuronal networks. Physica D, 118, 343-370.

Cohen, M. A., \& Grossberg, S. (1983). Absolute stability of global pattern formation and parallel memory storage by competitive neural networks. IEEE Trans. on Systems, Man, and Cybernetics, 13, 815-823.

Collins, J. J., Carson, C. C., Capela, A. C., \& Imhoff, T. T. (1996). Aperiodic stochastic resonance. Physical Review E, 54, 5575-5584.

Connors, B. W., \& Gutnick, M. J. (1990). Intrinsic firing patterns of diverse cortical neurons. Trends in Neurosci., 13, 99-104.

Cowan, J. D. (1968). Statistical mechanics of nervous nets. In E. R. Caianello (Ed.), Proceedings of the 1967 NATO Conference on Neural Networks. Berlin: Springer-Verlag.

Cox, D. R. (1962) Renewal theory. London: Methuen.

Cox, D. R., \& Lewis, P. A. W. (1966). The statistical analysis of series of events. London: Methuen.

Eggert, J., \& van Hemmen, J. L. Derivation of pool dynamics from microscopic neuronal models. In W. Gerstner et al. (Eds.), Artificial neural networksICANN'97 (pp. 109-114). Heidelberg: Springer-Verlag.

Ermentrout, G. B. (1981). N:M phase-locking in weakly coupled oscillators. J. Math. Biology, 12, 327-342.

Ernst, U., Pawelzik, K., \& Geisel, T. (1994). Multiple phase clustering of globally coupled neurons with delay. In M. Marinaro \& P. G. Morasso (Eds.), Proceedings of the ICANN'94 (pp. 1063-1065). Berlin: SpringerVerlag.

Feldman, J. L., \& Cowan, J. D. (1975). Large-scale activity in neural nets I: Theory with application to motoneuron pool responses. Biol. Cybern., 17, 29-38.

Fetz, E. E., \& Gustafsson, B. (1983). Relation between shapes of post-synaptic potentials and changes in firing probability of cat motoneurones. J. Physiol., 341, 387-410.

Gerstner, W. (1993). Kodierung und Signalübertragung in Neuronalen Systemen: Assoziative Netzwerke mit stochastisch feuernden Neuronen, Frankfurt/Main: Harri-Deutsch Verlag.

Gerstner, W. (1995). Time structure of the activity in neural network models. Phys. Rev. E, 51(1), 738-758.

Gerstner, W., \& van Hemmen, J. L. (1992). Associative memory in a network of "spiking" neurons. Network, 3, 139-164.

Gerstner, W., \& van Hemmen, J. L. (1993). Coherence and incoherence in a globally coupled ensemble of pulse emitting units. Phys. Rev. Lett., 71(3), 312-315.

Gerstner, W., \& van Hemmen, J. L. (1994). Coding and information processing in neural networks. In E. Domany, J. L. van Hemmen, \& K. Schulten (Eds.), Models of neural networks II (pp. 1-93). New York: Springer-Verlag.

Gerstner, W., van Hemmen, J. L., \& Cowan, J. D. (1996). What matters in neuronal 
locking. Neural Comput., 8, 1689-1712.

Golomb, D., Hansel, D., Shraiman, B., and Sompolinsky, H. (1992). Clustering in globally coupled phase oscillators. Phys. Rev. A, 45, 3516-3530.

Golomb, D., \& Rinzel, J. (1994). Clustering in globally coupled inhibitory neurons. Physica $D, 72,259-282$.

Gutkin, B., \& Ermentrout, G. G. (1998). Dynamics of membrane excitability determine inter-spike interval variability: A link between spike generation mechanism and cortical spike train statistics. Neural Comput., 10, 1047-1065.

Hansel, D., Mato, G., \& Meunier, C. (1995). Synchrony in excitatory neural networks. Neural Comput., 7, 307-337.

Hopfield, J. J. (1984). Neurons with graded response have computational properties like those of two-state neurons. Proc. Natl. Acad. Sci. USA, 81, 3088-3092.

Hopfield, J. J., \& Herz, A. V. M. (1995). Rapid local synchronization of action potentials: Towards computation with coupled integrate-and-fire networks. Proc. Natl. Acad. Sci. USA, 92, 6655.

Horn, D., and Levanda, S. (1998). Fast temporal encoding and decoding with spiking neurons. Neural Comput., 10, 1705-1720.

Hubel, D. H., \& Wiesel, T. N. (1962). Receptive fields, binocular interaction and functional architecture in the cat's visual cortex. J. Physiol. (London), 160, 106154.

Kandel, E. C., \& Schwartz, J. H. (1991). Principles of neural science. New York: Elsevier.

Kistler, W., Gerstner, W., \& van Hemmen, J. L. (1997). Reduction of HodgkinHuxley equations to a single-variable threshold model. Neural Comput., 9, 1015-1045.

Knight, B. W. (1972a). Dynamics of encoding in a population of neurons J. Gen. Physiology, 59, 734-766.

Knight, B. W. (1972b). The relationship between the firing rate of a single neuron and the level of activity in a population of neurons J. Gen. Physiology, 59, 767788.

Knox, C. K. (1974). Cross-correlation functions for a neuronal model Biophysical J., 14, 567-582.

Koch, C., Rapp, M., \& Segev, I. (1996). A brief history of time constants. Cerebral Cortex, 6, 93-101.

Kuramoto, Y. (1975). Self-entrainment of a population of coupled nonlinear oscillators. In H. Araki (Ed.), International Symposium on Mathematical Problems in Theoretical Physics (pp. 420-422). Berlin: Springer-Verlag.

Kuramoto, Y. (1984). Chemical oscillations, waves, and turbulence. Berlin: SpringerVerlag.

Kuramoto, Y. (1991). Collective synchronization of pulse-coupled oscillators and excitable units Physica D, 50, 15-30.

Mirollo, R. E., \& Strogatz, S. H. (1990). Synchronization of pulse coupled biological oscillators. SIAM J. Appl. Math., 50, 1645-1662.

Mountcastle, V. B. (1957). Modality and topographic properties of single neurons of cat's somatosensory cortex. J. Neurophysiol., 20, 408-434.

Nykamp, D., Tranchina, D., Shapley, R., \& McLoughlin, D. (1998). A population density approach facilitates large-scale modeling of neural networks. To 
appear in Journal of Computational Neuroscience.

Perkel, D. H., Gerstein, G. L., \& Moore, G. P. (1967). Neuronal spike trains and stochastic point processes. (I) the single spike train. Biophys. J., 7, 391418.

Pham, J., Pakdamen, K., Champagnat, J., \& Vibert, J.-R. (1998). Activity in sparsely connected excitatory neural networks: Effect of connectivity. Neural Networks, 11, 415-438.

Pinto, D. J., Brumberg, J. C., Simons, D. J., \& Ermentrout, G. B. (1996). A quantitative population model of whiskers barrels: Re-examining the Wilson-Cowan equations. J. Comput. Neurosci., 3, 247-264.

Plesser, H. E., \& Gerstner, W. (1999). Noise in integrate-and-fire neurons: From stochastic input to escape rates. To appear in Neural Computation

Poliakov, A. V., Powers, R. K., \& Binder, M. D. (1997). Functional identification of the input-output transforms of motoneurons in the rat and the cat $J$. Physiology, 504, 2:401-424.

Senn, W., Wyler, K., Streit, J., Larkum, M., Lüscher, H.-R., Mey, H., Müller, L., Steinhauser, D., Vogt, K., \& Wannier, T. (1996). Dynamics of random neural network with synaptic depression. Neural Networks, 9, 575-588.

Somers, D., \& Kopell, N. (1993). Rapid synchronization through fast threshold modulation. Biol. Cybern., 68, 393-407.

Spiridon, M., Chow, C. C., \& Gerstner, W. (1998). Frequency spectrum of coupled stochastic neurons with refractoriness. In L. Niklasson et al. (Eds.), ICANN'98 (pp. 337-342). Berlin: Springer-Verlag.

Stein, R. B. (1967). Some models of neuronal variability. Biophys. J., 7, 37-68.

Stevens, C. F., \& Zador, A. (1998). Novel integrate-and-fire-like model of repetitive firing in cortical neurons. In Proceedings of the Fifth Joint Symposion on Neural Computation, University of California at San Diego.

Strogatz, S. H., \& Mirollo, R. E. (1991). Stability of incoherence in a population of coupled oscillators. J. Stat. Phys., 63, 613-635.

Terman, D., \& Wang, D. (1995). Global competition and local cooperation in a network of neural oscillators. Physica D, 81, 148-176.

Thorpe, S., Fize, D., \& Marlot, C. (1996). Speed of processing in the human visual system. Nature, 381, 520-522.

Treves, A. (1992). Local neocortical processing: A time for recognition, Int. J. Neural Systems, 3(Supp.), 115-119.

Treves, A. (1993). Mean-field analysis of neuronal spike dynamics. Network, 4 , 259-284.

Treves, A., Rolls, E. T., \& Simmen, M. (1997). Time for retrieval in recurrent associative memories. Physica D, 107, 392-400.

Tsodyks, M., Mitkov, I., \& Sompolinsky, H. (1993). Patterns of synchrony in inhomogeneous networks of oscillators with pulse interaction. Phys. Rev. Lett., 71, 1281-1283.

Tsodyks, M. V., \& Sejnowski, T. (1995). Rapid state switching in balanced cortical networks. Network, 6, 111-124.

Tuckwell, H. C. (1988). Introduction to theoretic neurobiology. Cambridge: Cambridge University Press.

Tuckwell, H. C. (1989). Stochastic processes in the neurosciences. Philadelphia: 
SIAM.

van Vreeswijk, C., Abbott, L. E., \& Ermentrout, G. B. (1994). When inhibition not excitation synchronizes neural firing. Journal of Computational Neuroscience, 1 , 313-321.

van Vreeswijk, C., \& Sompolinsky, H. (1996). Chaos in neuronal networks with balanced excitatory and inhibitory activity. Science, 274, 1724-1726.

Wilson, H. R., \& Cowan, J. D. (1972). Excitatory and inhibitory interactions in localized populations of model neurons. Biophysical J., 12, 1-24.

Wilson, H. R., \& Cowan, J. D. (1973). A mathematical theory of the functional dynamics of cortical and thalamic nervous tissue. Kybernetik, 13, 55-80.

Winfree, A. T. (1980). The geometry of biological time. Berlin: Springer-Verlag.

Received March 23, 1998; accepted January 13, 1999. 